\title{
Endothelial deficiency of L1 reduces tumor angiogenesis and promotes vessel normalization
}

\author{
Elena Magrini, ${ }^{1}$ Alessandra Villa, ${ }^{1,2}$ Francesca Angiolini, ${ }^{1,2}$ Andrea Doni, ${ }^{3}$ Giovanni Mazzarol, ${ }^{2}$ Noemi Rudini, ${ }^{4}$ Luigi Maddaluno, ${ }^{4}$ \\ Mina Komuta, ${ }^{5}$ Baki Topal, ${ }^{6}$ Hans Prenen, ${ }^{7}$ Melitta Schachner, ${ }^{8}$ Stefano Confalonieri, ${ }^{2}$ Elisabetta Dejana, ${ }^{4}$ Fabrizio Bianchi, ${ }^{1,2}$ \\ Massimiliano Mazzone, ${ }^{9,10}$ and Ugo Cavallaro ${ }^{1,2}$ \\ DDepartment of Experimental Oncology and 2Molecular Medicine Program, European Institute of Oncology, Milano, Italy. ${ }^{3}$ Department of Inflammation and Immunology, Humanitas Clinical and \\ Research Center, Rozzano, Milano, Italy. ${ }^{4}$ IFOM, FIRC Institute of Molecular Oncology, Milano, Italy. ${ }^{5}$ Department of Morphology and Molecular Pathology, ${ }^{6}$ Department of Abdominal Surgery, and \\ ${ }^{7}$ Department of Gastroenterology, University Hospitals Leuven, Leuven, Belgium. ${ }^{8}$ W.M. Keck Center for Collaborative Neuroscience and Department of Cell Biology and Neuroscience, \\ Rutgers University, Piscataway, New Jersey, USA. 'Laboratory of Molecular Oncology and Angiogenesis, Vesalius Research Center, Vlaams Instituut voor Biotechnologie (VIB), \\ Leuven, Belgium. ${ }^{10}$ Department of Oncology, Katholieke Universiteit (KU) Leuven, Leuven, Belgium.
}

While tumor blood vessels share many characteristics with normal vasculature, they also exhibit morphological and functional aberrancies. For example, the neural adhesion molecule L1, which mediates neurite outgrowth, fasciculation, and pathfinding, is expressed on tumor vasculature. Here, using an orthotopic mouse model of pancreatic carcinoma, we evaluated L1 functionality in cancer vessels. Tumor-bearing mice specifically lacking L1 in endothelial cells or treated with anti-L1 antibodies exhibited decreased angiogenesis and improved vascular stabilization, leading to reduced tumor growth and metastasis. In line with these dramatic effects of L1 on tumor vasculature, the ectopic expression of L1 in cultured endothelial cells (ECs) promoted phenotypical and functional alterations, including proliferation, migration, tubulogenesis, enhanced vascular permeability, and endothelial-to-mesenchymal transition. L1 induced global changes in the EC transcriptome, altering several regulatory networks that underlie endothelial pathophysiology, including JAK/STAT-mediated pathways. In particular, L1 induced IL-6-mediated STAT3 phosphorylation, and inhibition of the IL-6/JAK/STAT signaling axis prevented L1-induced EC proliferation and migration. Evaluation of patient samples revealed that, compared with that in noncancerous tissue, L1 expression is specifically enhanced in blood vessels of human pancreatic carcinomas and in vessels of other tumor types. Together, these data indicate that endothelial L1 orchestrates multiple cancer vessel functions and represents a potential target for tumor vascular-specific therapies.

\section{Introduction}

Cancer growth strictly depends on the expansion of the host vasculature, which not only supplies oxygen and nutrients to the tumor tissue, but also provides cancer cells with the metastatic route to colonize distant organs. Therefore, angiogenesis represents a critical process during tumor initiation and malignant progression (1). Different strategies have been developed to reduce angiogenesis and thus control tumor progression, as exemplified by the inhibition of VEGF-dependent pathways. However, while some cancer types show a certain degree of therapeutic response, the benefits of such antiangiogenic agents are transient and the initial response is often followed by the establishment of resistance and escape mechanisms, leading to tumor relapse $(2,3)$. This highlights the need for a more comprehensive understanding of the biological processes that underlie tumor vascularization, which, in turn, would set the stage for additional angiogenesistargeted therapies.

Authorship note: Alessandra Villa and Francesca Angiolini contributed equally to this work

Conflict of interest: The authors have declared that no conflict of interest exists. Submitted: April 26, 2013; Accepted: July 17, 2014.

Reference information: / Clin Invest. 2014;124(10):4335-4350. doi:10.1172/JCI70683.
Compared with their normal counterparts, tumor vessels are aberrant in almost all aspects of their structure and function. They are heterogeneous and tortuous, branch chaotically, and have an uneven vessel lumen. In addition, they frequently lack pericyte coverage and show an abnormal basement membrane, resulting in vascular instability and altered permeability. These vessel abnormalities generate a promalignant microenvironment, characterized by hypoxia, low $\mathrm{pH}$, and high fluid pressure, which can select for more malignant cancer cells and facilitate their dissemination through leaky vessels, thus causing poor response to therapy (4). These findings raise the question of whether tumor vessel normalization provides an alternative therapeutic opportunity in order to reduce metastatic spread and enhance tumor responses to chemotherapy and radiotherapy (4). However, the characteristics that make tumor-associated endothelial cells (ECs) different from normal ECs are not yet fully identified, and their properties are usually extrapolated from the behavior of ECs during vascular development. Moreover, while it is well known that different and morphologically distinct EC types, such as tip, stalk, and phalanx cells, coexist during normal vascularization, the heterogeneity of cancer-associated ECs is still poorly understood. Hence, a deeper understanding of the phenotypical heterogeneity and specific molecular signature of tumor vasculature is essential to elucidat- 
A
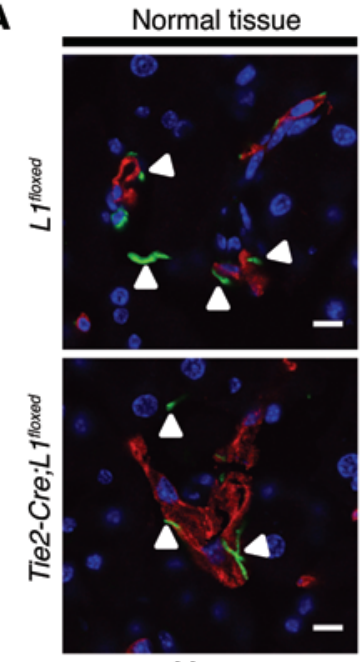

Merge

B
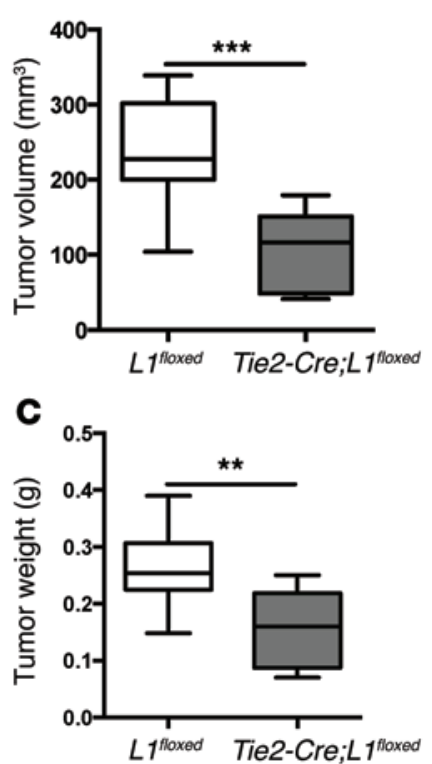
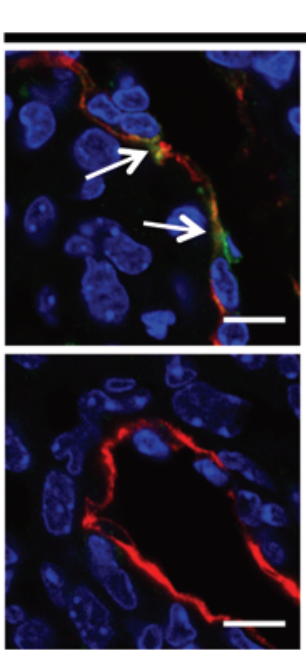

Merge

D

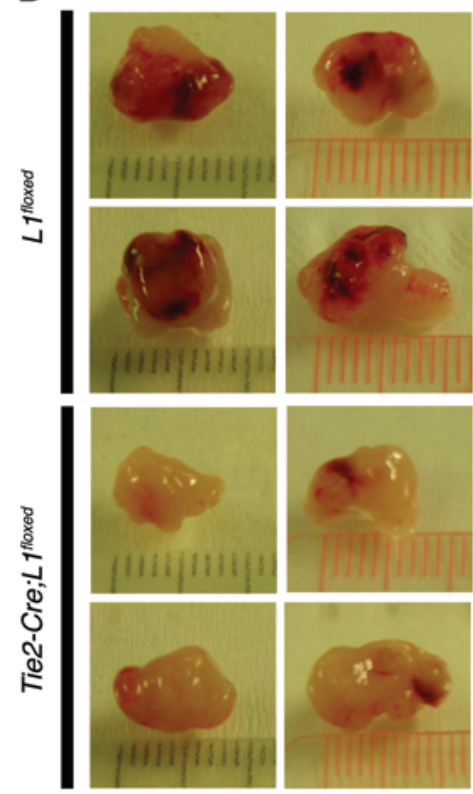

Tumor

PECAM-1
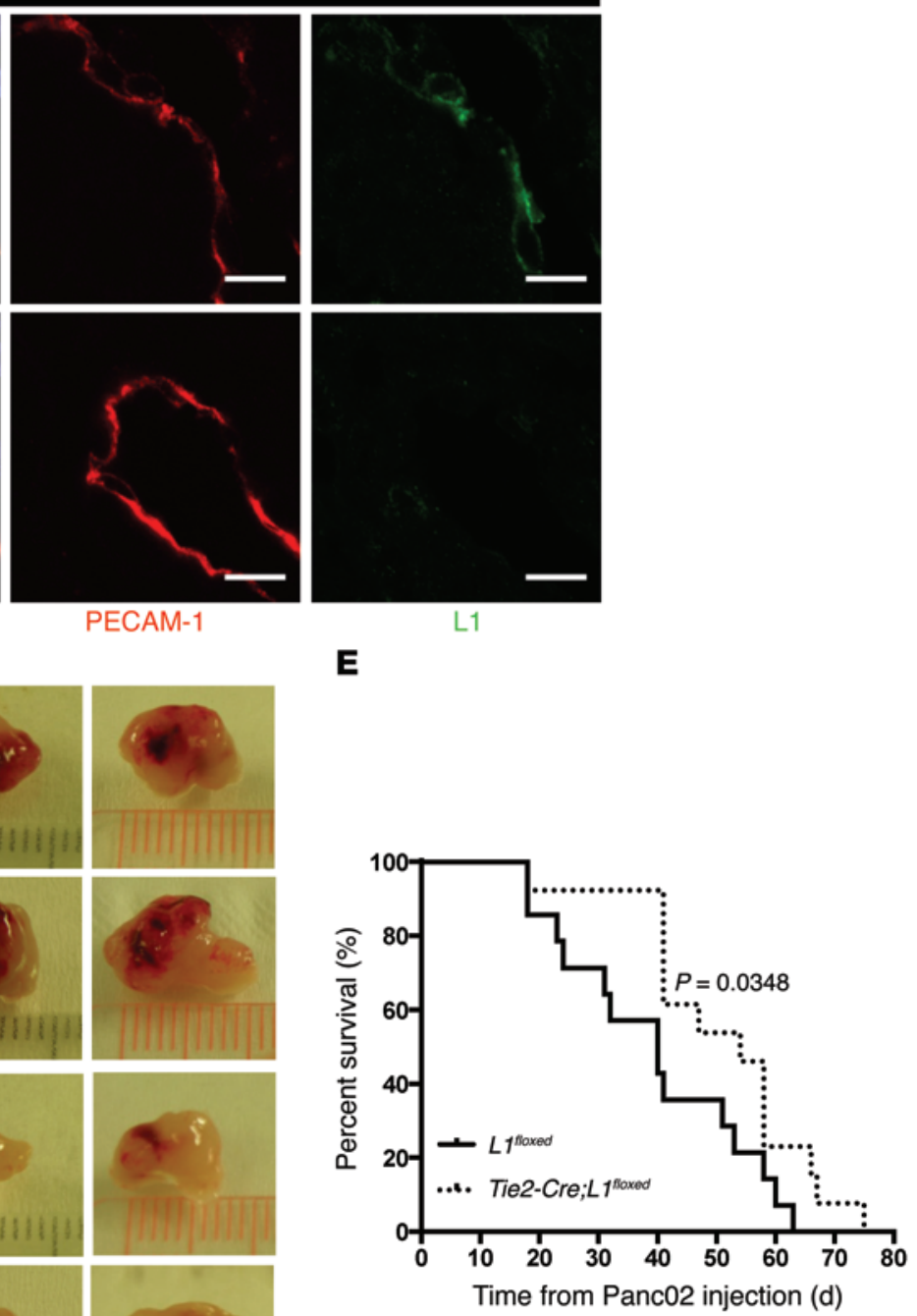

Figure 1. Ablation of endothelial L1 in Tie2-Cre; L 1 floxed mice and related effects on tumor development and mouse survival. (A) Sections of normal pan-

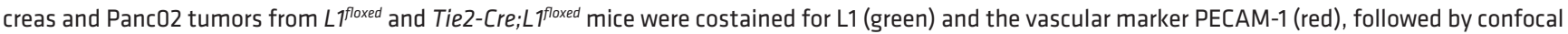
analysis. Arrows indicate vessels coexpressing PECAM-1 and L1; arrowheads indicate L1-positive nerves that served as internal control. Scale bars: $10 \mu \mathrm{m}$. (B and C) The volume (B) and weight (C) of pancreatic tumors from $L 7^{\text {floxed }}$ and Tie2-Cre; $L$ floxed $^{\text {fice }}$ were recorded 14 days after Panc02 injection. Data rep-

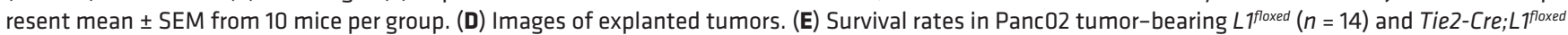
mice $(n=13) .{ }^{* *} P<0.01 ;{ }^{* *} P<0.001$.

ing the mechanisms of pathological angiogenesis and to identifying novel cancer vessel-specific markers.

L1 (also known as L1CAM or CD171) is a transmembrane glycoprotein belonging to the immunoglobulin superfamily and is composed of an extracellular portion, containing 6 Ig-like domains and 5 fibronectin type III repeats, followed by a transmembrane region, and a highly conserved cytoplasmic tail (5). L1 was discovered and characterized as a cell-adhesion molecule in the nervous system (6), where it is involved in neurite outgrowth and fasciculation as well as cell adhesion and migration. In addition to homophilic binding, L1 can establish cis- or trans-interactions with different binding partners, such as integrins, CD24, neurocan, neuropilin-1, and other members of the neural cell adhesion family (7).
Besides the nervous system, L1 is expressed in many human cancers, including ovarian and endometrial carcinoma, pancreatic ductal adenocarcinoma (PDAC), melanoma and glioblastoma. L1 expression confers motile and invasive properties to tumor cells, supporting cancer growth, metastasis, and chemoresistance and often acting as a marker of poor prognosis (8). L1 has also been detected in the hematopoietic system, in particular in immune cells of myelomonocytic and lymphoid origin (9), and we have previously reported L1-dependent transmigration of dendritic cells across the endothelium (10).

An intriguing aspect of L1 biology is its expression in the vascular system: while no or very little L1 is detectable in the vasculature of most normal tissues, its level is markedly increased in the vas- 


\section{Table 1. Number of mice with metastases and number of site- specific metastases in Panc02 tumor-bearing L $1^{\text {floxed }}$ and Tie2- Cre; $L 1^{\text {floxed }}$ mice}

$\begin{array}{lcc}\text { Mice with metastasis } & \begin{array}{c}\text { Lfloxed } \\ 5 / 7(71 \%)\end{array} & \begin{array}{c}\text { Tie2-Cre; } L \text { floxed } \\ 2 / 7(29 \%)\end{array} \\ \begin{array}{lcc}\text { Site-specific metastases (number of nodules) } \\ \text { Stomach }\end{array} & 1 & 0 \\ \text { Intestine } & 10 & 0 \\ \text { Liver } & 1 & 1 \\ \text { Kidney } & 3 & 0 \\ \text { Urogenital tract } & 1 & 0 \\ \text { Diaphragm } & 3 & 3 \\ \text { Abdominal wall } & 11 & 8\end{array}$

$n=7$ mice per group.

cular endothelium associated with pathological conditions, most prominently cancer and inflammatory diseases. Furthermore, various cytokines and angiogenic growth factors abundantly released in the tumor microenvironment, such as VEGF-A, TNF- $\alpha$, IFN- $\gamma$, and TGF- $\beta 1$, upregulate L1 expression in ECs $(10,11)$. Taken together, these observations point to $\mathrm{L} 1$ as a tumor vessel-specific molecule. However, it remains elusive whether L1 is causally involved in the formation and/or function of cancer-associated vasculature.

In this study, we investigated the role of vascular L1 in a mouse model of pancreatic tumor in which the endothelial-specific ablation of L1 was achieved by Cre-LoxP technology. This approach was complemented by in vitro studies on ECs in which the expression of L1 was manipulated in order to induce overexpression or silencing of the gene. Our data revealed that L1 plays a pivotal role in tumor-associated vessels, thus influencing cancer growth, metastasis, and mouse survival. In particular, L1 was found to modulate different EC functions and to promote endothelial-tomesenchymal transition (EndMT), a process causally linked to neovascularization (12). We also demonstrated that antibodymediated targeting of $\mathrm{L} 1 \mathrm{in}$ tumor-bearing mice leads to decreased neovascularization and to vessel normalization, resulting in reduced tumor growth. Finally, we obtained mechanistic insights into the function of L1 in ECs by showing that (a) L1 modulates several transcriptional programs in ECs, including pathways that govern vascular development and function; and (b) L1 promotes the activation of the IL-6/JAK/STAT signaling pathway, and the latter mediates L1-induced stimulation of ECs.

\section{Results}

Endothelial $L 1$ regulates pancreatic tumor growth and metastasis in mice. To define the role of vascular L1 in tumor growth, we combined the genetic inactivation of endothelial L1 with an orthotopic mouse model of pancreatic carcinoma. This model is based on the injection of mouse Panc02 cells into the head of the pancreas of syngeneic C57BL/6 mice, resulting in the formation of highly vascularized and metastatic pancreatic tumors (13). Panc02 cells do not express L1, either in culture (Supplemental Figure 1, A and B; supplemental material available online with this article; doi:10.1172/ JCI70683DS1) or upon tumor formation in mice (not shown). The endothelial-specific ablation of L1 in mice was achieved by crossing Tie2-Cre with $L 1^{\text {floxed }}$ mice (10). Indeed, L1 was readily detectable in the tumor vessels of control, $L 1^{\text {floxed }}$ mice, while no L1 immunoreactivity was observed in the vasculature of Tie2-Cre;L1 $1^{\text {foxed }}$ tumors (Figure 1A). Interestingly, the vessels of normal pancreatic tissue in either L1 ${ }^{\text {floxed }}$ or Tie2-Cre; L1 $^{\text {floxed }}$ mice showed no L1 expression (Figure 1A). As expected, L1 expression in peripheral nerves was not affected by Tie2-Cre-mediated recombination, thus acting as an internal control (Figure 1A and Supplemental Figure 2).

Given that the Tie2 gene promoter is also active in hematopoietic precursors and $\mathrm{L} 1$ is expressed in certain immune cell lineages (10), we first checked whether Tie2-Cre-mediated ablation of L1 affected immune cell infiltration into the tumor. No difference in the number of tumor-infiltrating immune cells, assessed either as total $\mathrm{CD} 45^{+}$leukocytes or as individual hematopoietic cell subpopulations identified by lineage-specific markers, was observed between control and Tie2-Cre;L1 $1^{\text {floxed }}$ mice (Supplemental Figure 3 , A and B). In agreement with our previous results (10), the ablation of $\mathrm{L} 1$ in $\mathrm{Tie} 2^{+}$hematopoietic progenitors did not cause major defects in mouse hematopoiesis, as blood cell counts for the different leukocyte populations gave very similar values for $L 1^{\text {foxed }}$ and Tie2-Cre;L1 ${ }^{\text {floxed }}$ littermates (Supplemental Figure 3C). Overall, these data indicate that in the Panc02 tumor model, L1 deficiency does not affect immune cell migration into cancer tissue and, therefore, any effect on tumor behavior upon Tie2 promoter-driven ablation of L1 in Tie2-Cre;L1floxed mice should be attributed to endothelial L1 rather than to L1 expressed in immune cells.

We then analyzed Panc02 tumors 14 days after implantation. Pancreatic tumor burden was markedly reduced in Tie2-Cre;L1 $1^{\text {foxed }}$ mice (Figure 1, B and C), implicating vascular L1 in cancer growth. This effect was accompanied by an increase in TUNEL-positive apoptotic cells in Tie2-Cre; $L 1^{\text {floxed }}$ tumors (Supplemental Figure 4A), while cancer cell proliferation, as assessed by staining for either Ki-67 or phospho-histone H3, was not affected (Supplemental Figure 4, B and C). These data indicated that vascular L1 deficiency is associated with reduced tumor growth and increased apoptosis.

A macroscopical examination revealed that tumors of $L 1^{\text {floxed }}$ mice exhibited intensely red areas, suggesting high tumor vascularization and/or hemorrhages. Notably, this feature was greatly reduced in

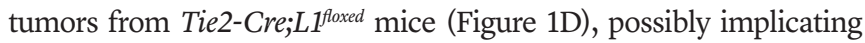
endothelial L1 in tumor angiogenesis and/or vascular permeability.

Vascular L1 also appeared to be involved in the dissemination of Panc02 tumors, since only $29 \%$ of Tie2-Cre; L1 $^{\text {foxed }}$ mice $(2 / 7)$ showed metastatic lesions in different abdominal organs, while metastases were detected in $71 \%$ of $L 1^{\text {floxed }}$ mice $(5 / 7)$ (Table 1 ). Consistent with the reduced cancer growth and dissemination, tumor-bearing Tie2-Cre; L1 foxed mice also showed longer survival times than their control littermates (Figure 1E). Overall, these results indicated that the ablation of endothelial L1 results in decreased tumor malignancy.

Endothelial $L 1$ regulates tumor angiogenesis and vascular normalization. To determine whether endothelial L1 plays any role in cancer vascularization, we measured the microvessel density in tumor tissue. Panc02 tumors in Tie2-Cre; L1 $^{\text {floxed }}$ mice exhibited a reduced number of vessels as compared with $L 1^{\text {floxed }}$ mice, indicating that endothelial L1 is involved in tumor angiogenesis (Figure 2, A and B). These findings were further supported by in vivo 
A
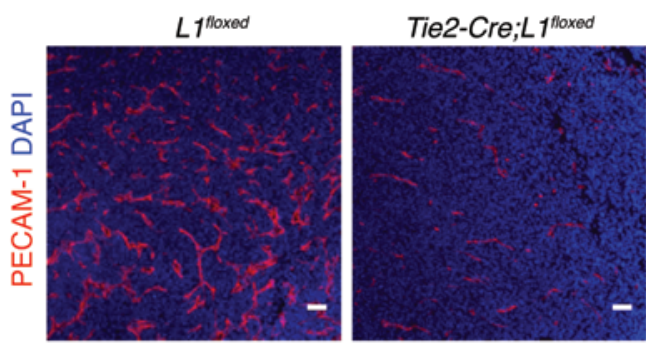

C
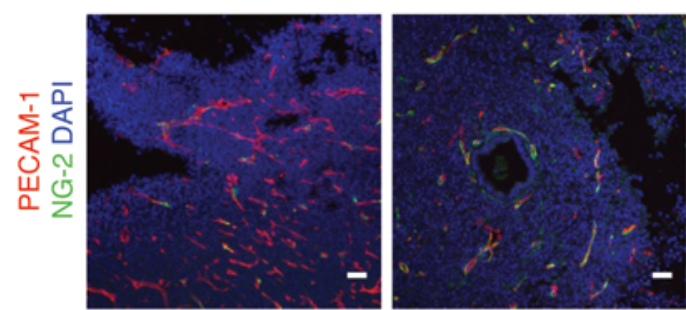

E

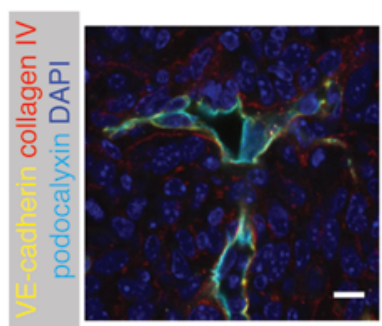

G

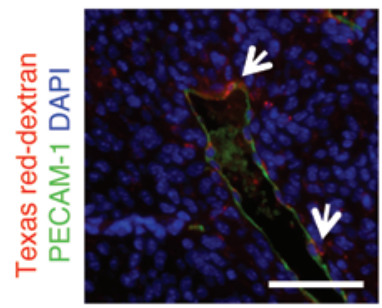

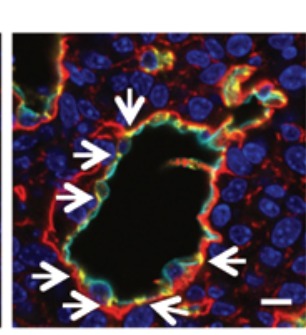

F

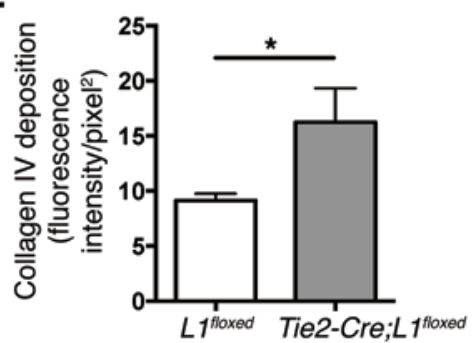

H

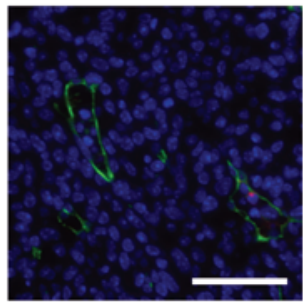

B

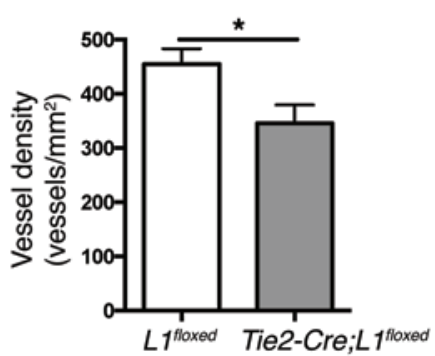

D
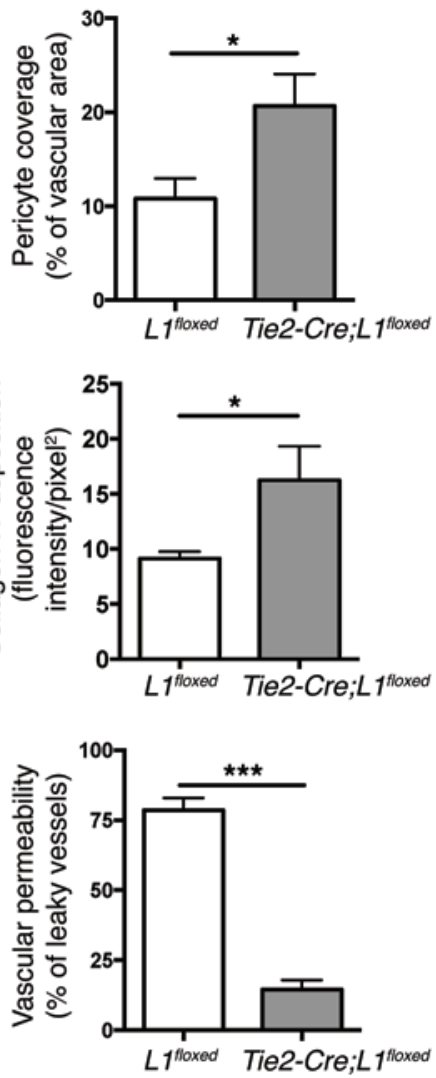

Figure 2. Endothelial L1 deficiency results in reduced tumor angiogenesis and in vessel normalization. (A) Representative images of Panc02 tumor sections stained for PECAM-1 (red) to visualize vessels. Scale bars: $50 \mu \mathrm{m}$. (B) Quantitation of vessel density in tumors from $L 7^{\text {floxed }}(n=9)$ and Tiez-Cre; $L 1^{\text {floxed }}$ mice $(n=5)$. (C) Representative images of Panc02 tumor sections costained for PECAM-1 (red) and the pericyte marker NG-2 (green) to visualize pericyte coverage. Scale bars: $50 \mu \mathrm{m}$. (D) Quantitation of pericyte coverage in tumor vessels from $L$ ffloxed $^{(}(n=8)$ and Tie2-Cre; L floxed mice $(n=5)$. (E) Representative images of Panc02 tumor sections costained for the endothelial apical marker podocalyxin (cyan), the junctional marker VE-cadherin (yellow), and the basement membrane marker collagen IV (red). Arrows indicate the localization of VE-cadherin at cell-cell contact in Tie2-Cre; L $7^{\text {floxed }}$ tumor vessels (right), which is lost or dramatically reduced in $L f^{\text {floxed }}$ vessels (left). Scale bars: $10 \mu \mathrm{m}$. (F) Quantitation of collagen IV deposition in tumor vessels from $L 7^{\text {floxed }}$ $(n=3)$ and Tie2-Cre; L $7^{\text {floxed }}$ mice $(n=3)$. (G) Representative images of Panc02 tumor sections from mice injected with Texas red-dextran. Sections were costained for Texas red (red) and PECAM-1 (green). Scale bars: $50 \mu \mathrm{m}$. (H) Quantitation of vascular permeability, expressed as the percentage of vessels showing extravasated dextran in tumors from $L 7^{\text {floxed }}$ $(n=3)$ and Tie2-Cre; L $7^{\text {floxed }}$ mice $(n=3)$. ${ }^{*} P<0.05 ;{ }^{* * *} P<0.0005$.
Matrigel plug assays, where Tie2-Cre;L1 $1^{\text {floxed }}$ mice showed a marked decrease in the neovascularization induced by the angiogenic factor FGF-2 (Supplemental Figure 5).

We also tested the hypothesis that $\mathrm{L} 1$ regulates vascular integrity in cancer tissue. To this goal, given that the interaction of pericytes with the endothelium is causally linked to vessel stabilization and integrity (14), we analyzed pericyte coverage in the vasculature of $\mathrm{Panc0} 2$ tumors. The extent of pericyte coverage, as assessed by costaining for PECAM- 1 and the pericyte marker NG-2 (15), was significantly increased in Tie2-Cre; $L 1^{\text {floxed }}$ mice (Figure 2, C and D), thus implying that endothelial L1 negatively regulates the recruitment of pericytes to the vascular wall.

Next, we asked whether endothelial polarity, another prerequisite for vessel integrity, was affected by L1. Panc02 tumors were stained for collagen IV, a major constituent of the vascular basement membrane that is essential for capillary stability and organization (16), for the apical marker podocalyxin and for VE-cadherin as a marker of endothelial junctions (17). In line with the notion that the architecture of cancer-associated vasculature is irregular and disorganized (4), the tumors of $L 1^{\text {floxed }}$ mice showed a dramatic disruption of endothelial polarity, with no or very low level of collagen IV, the irregular distribution of podocalyxin within the vessel wall, and a lack of VE-cadherin-marked junctions (Figure 2E). In contrast, the vasculature of Tie2-Cre; $L 1^{\text {floxed }}$ tumors displayed abundant deposition of collagen IV in the basement membrane (Figure 2, E and F), localization of podocalyxin at the luminal surface, and a regular pattern of VE-cadherin localization, consistent with its accumulation at cell-cell contacts (Figure 2E). These findings implicated L1 in the maintenance of endothelial polarity.

Vascular abnormalities such as defective pericyte coverage, altered polarity, and disorganized endothelial junctions, lead to disruption of endothelial barrier function and, hence, to vascular leakage, thus implying that L1 might regulate vascular permeability. To test this hypothesis, we assessed the extravasation of intravenously injected 40-kDa dextran in Panc02 tumors. Indeed, tumor vascular permeability to dextran was remarkably higher in control, L1 $1^{\text {floxed }}$ mice as compared with Tie2-Cre;L1 $1^{\text {foxed }}$ mice (Figure 2, $\mathrm{G}$ and $\mathrm{H}$ ), thus implicating $\mathrm{L} 1$ in tumor vessel leakiness.

Taken together, these observations point to endothelial L1 as a causal player in enhanced tumor angiogenesis as well as in the dis- 


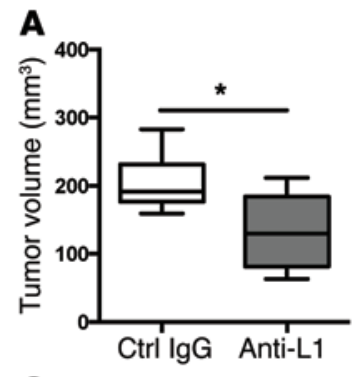

C Ctrl lgG
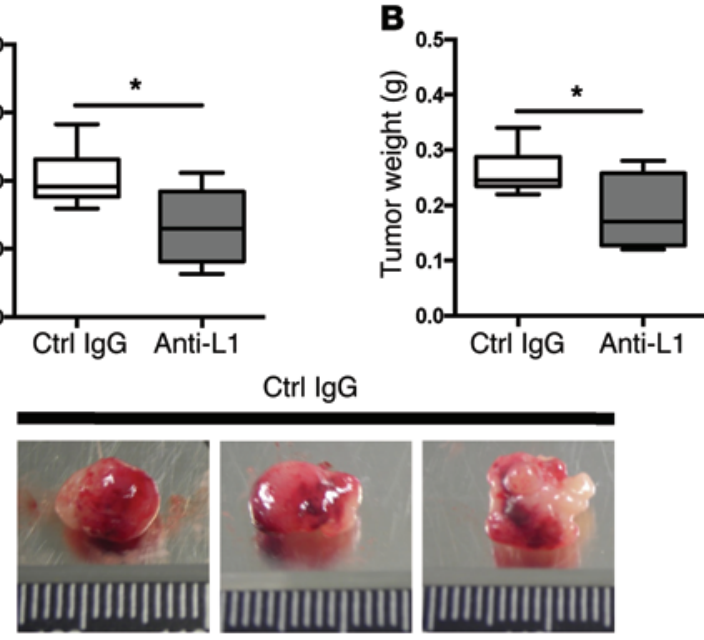

Anti-L1

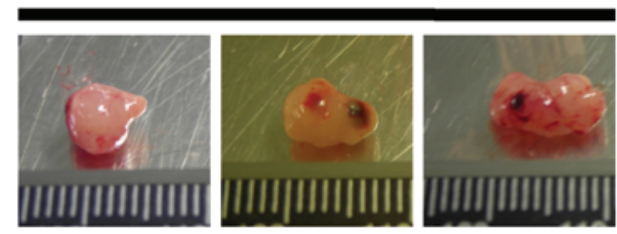

D

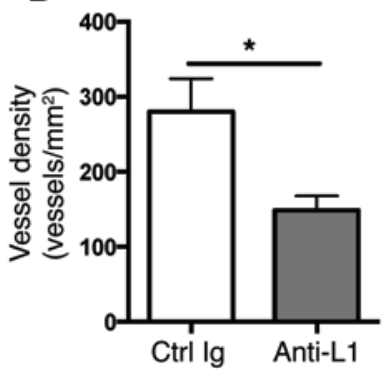

E

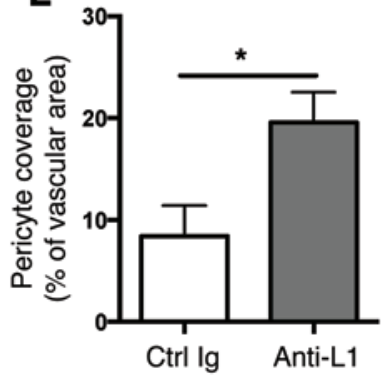

ruption of vascular integrity and stability that is commonly associated with cancer development.

In vivo targeting of $L 1$ reduces tumor growth and angiogenesis and promotes vessel normalization. The results obtained with the genetic ablation of L1 in Panc02 tumor-bearing mice implied that L1 might be a therapeutic target for preventing cancerassociated neovascularization and/or enhancing vessel normalization. To explore this possibility, we generated a polyclonal antibody against the ectodomain of mouse L1. First, we tested the function-blocking activity of the antibody in vitro. ECs were transfected with L1 and then subjected to functional assays in the presence of the antibody. As described in more detail in the next section, L1 induced EC proliferation, migration, and tube formation, and the anti-L1 antibody inhibited all these activities (Supplemental Figure 6), thus confirming its neutralizing activity. The antibody was then used to target host L1 in the Panc02 mouse tumor model. As shown in Figure 3, A and B, tumor growth was significantly reduced in mice treated with the anti-L1 antibody as compared with mice treated with control antibody. Furthermore, the intensely red areas present in control tumors were markedly reduced in anti-L1-treated tumors (Figure 3C), in agreement with our observations in Tie2-Cre;L1 $1^{\text {floxed }}$ mice (Figure 1D). The anti-L1 antibody caused a significant decrease of vessel density in Panc02 tumors (Figure 3D), thus supporting the
Figure 3. Treatment with anti-L1 antibodies reduces tumor growth and angiogenesis while increasing pericyte coverage in tumor vessels. The volume (A) and weight (B) of pancreatic tumors from mice treated with anti-L1 antibodies $(n=6)$ or control (ctrl) IgG $(n=6)$ were recorded 14 days after Panc02 injection. Data represent means \pm SEM. (C) Images of explanted tumors. (D) Quantitation of vessel density in tumors from mice treated with anti-L1 antibodies $(n=5)$ or control IgG $(n=5)$. (E) Quantitation of pericyte coverage in tumor vessels from mice treated with anti-L1 antibodies $(n=6)$ or control IgG $(n=5)$. ${ }^{*} P<0.05$.

notion that targeting L1 might be a suitable anti-angiogenic strategy. Finally, tumor vessels from anti-L1-treated mice showed a higher rate of pericyte coverage than tumors treated with control antibody (Figure 3E).

Thus, the treatment of tumor-bearing mice with the anti-L1 antibody, besides confirming the result of genetically ablating endothelial L1, revealed that the pharmacological inactivation of L1 might represent a novel strategy to interfere with tumor neovascularization and to enhance vessel stabilization.

L1 orchestrates the angiogenic behavior of ECs. The reduced tumor microvessel density upon ablation of L1 in the endothelium prompted us to test whether L1 is involved in the critical EC processes that underlie angiogenesis, i.e., proliferation, migration, and tubulogenesis. Toward this goal, we employed immortalized mouse lung ECs (luECs) because, due to the expression of moderate levels of endogenous L1 (Supplemental Figure 7A), they were amenable to both gain- and loss-of-function studies. The luECs were stably transfected with the murine L1 cDNA (Supplemental Figure 7, A and B). As shown in Figure 4A, forced expression of L1 resulted in increased luEC proliferation. This result was confirmed in a classical in vivo assay for EC proliferation (18), where luECs were injected subcutaneously into immunodeficient mice and allowed to form hemangioma-like lesions. As shown in Figure 4B and C, hemangiomas formed by L1-transfected luECs exhibited a markedly higher growth rate than those formed by control luECs, consistent with our in vitro results on L1-induced proliferation of ECs. To further validate and extend these observations, we employed a loss-of-function approach. The knockdown of L1 with 2 different siRNAs (Supplemental Figure 8A) was accompanied by a decrease in luEC proliferation compared with control siRNAtransfected cells (Supplemental Figure 8B). In addition, L1 silencing also reduced the proliferative response of luECs to TNF- $\alpha$ stimulation (Supplemental Figure 8C). Thus, L1 plays a pivotal role in EC proliferation.

Next, a scratch-wound assay was used to evaluate the possible effect of L1 on EC migration. L1-transfected luECs showed a higher migration rate than control cells (Figure 4D and Supplemental Figure 7C). Conversely, $L 1$ gene silencing resulted in decreased luEC migration (Supplemental Figure 8, D and E). These data demonstrate that L1 is causally involved in EC migration.

We also assessed the effect of L1 in in vitro angiogenesis assays, determining the ability of ECs to form tube-like structures in $3 \mathrm{D}$ reconstituted extracellular matrix. As shown in Figure $4 \mathrm{E}$ and Supplemental Figure 7D, forced expression of L1 significantly enhanced luEC tube formation as compared with control ECs.

Thus, L1 regulates EC proliferation, migration, and tubulogenesis, considered to be key cellular processes during the angiogenic cascade. 
A
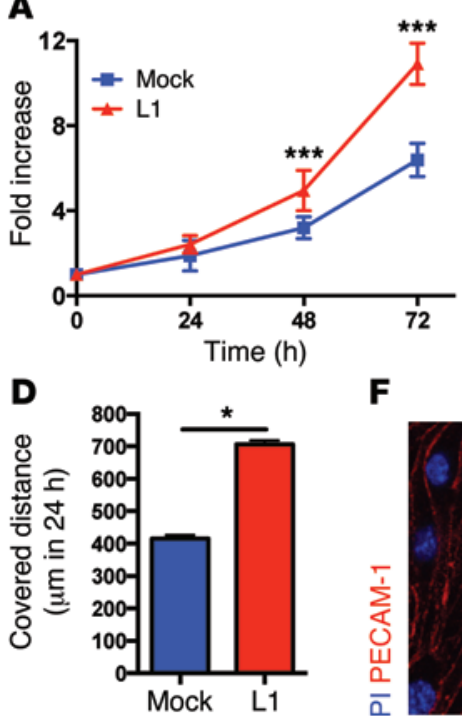

E

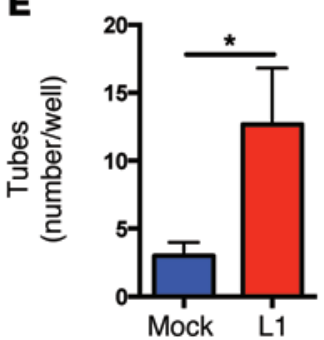

F
B
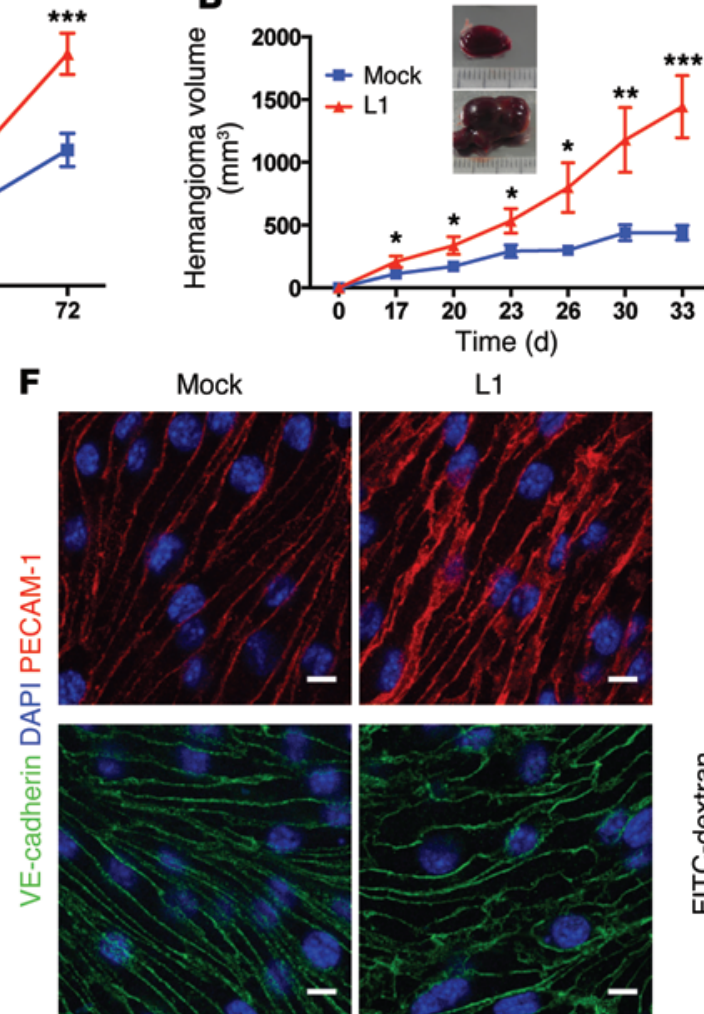

C

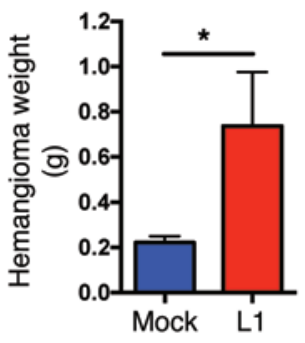

G
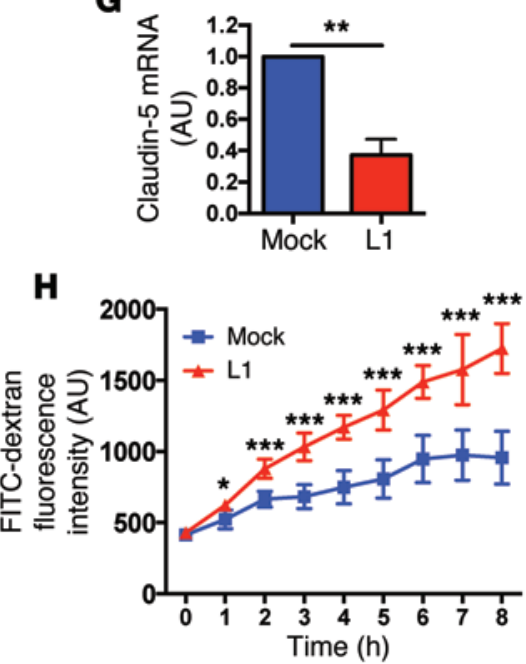

Figure 4. L1 confers an angiogenic phenotype to ECs and enhances endothelial permeability. (A) Proliferation curves of mock- and L1-transfected luECs. (B) Growth curves of hemangiomas formed by mock- or L1-transfected luECs injected subcutaneously into nude mice, as determined by volume measurement at the indicated time points. Representative images of hemangiomas explanted are shown (insets). (C) Weight of hemangiomas explanted 33 days after injection of mock- or L1-transfected luECs. (D) Migration assays of mock- and L1-transfected luECs were performed as described in Methods. (E) Matrigel-based tube formation assays of mock- and L1-transfected luECs were performed as described in Methods. (F) Mock- or L1-transfected luECs were stained for PECAM-1 (red) or VE-cadherin (green) prior to confocal analysis. Scale bars: $10 \mu \mathrm{m}$. (G) qRT-PCR analysis of claudin-5 mRNA in mock- and L1-transfected luECs. Transcript levels were normalized as described in Methods and are shown as fold changes in L1-transfected cells relative to mocktransfected cells $(n=3)$. (H) FITC-dextran permeability assays were performed on monolayers of mock- and L1-transfected luECs as described in Methods. Data in $\mathbf{A}, \mathbf{D}, \mathbf{E}$, and $\mathbf{H}$ represent the mean $\pm \mathrm{SD}$ from a representative experiment performed at least in triplicate. Data in $\mathbf{B}$ and $\mathbf{C}$ represent mean $\pm \mathrm{SEM}$ from 10 to 12 mice per group. ${ }^{*} P<0.05 ;{ }^{* *} P<0.01 ;{ }^{* * *} P<0.001$.

Our data on Tie2-Cre; If $^{\text {foxed }}$ mice indicated that $\mathrm{L} 1$ modulates the permeability of tumor vessels. Since vascular permeability is strictly related to the organization and function of cell-cell junctions in the endothelium, we determined whether L1 affected the expression and localization of junctional proteins in luECs. Staining for 2 prototypical adhesion molecules of the endothelium, PECAM-1 and VE-cadherin, showed that the architecture of cell-cell boundaries was disorganized in L1-transfected luECs (Figure 4F), despite the fact that the total level of either adhesion molecule was unaffected (Supplemental Figure 9A). This observation was consistent with the in vivo data showing that the altered localization of VE-cadherin in the vasculature of Panc02 tumors was reverted upon endothelial ablation of L1 in Tie2-Cre; L $^{\text {foxed }}$ mice (Figure 2E). We found no differences in the levels of other components of the interendothelial adhesion complexes, such as 4 catenin family members $(\alpha, \beta, \gamma$, and p120-catenin) and junctional adhesion molecule-A (Supplemental Figure 9B). We then tested whether $\mathrm{L} 1$ affected the expression of claudin-5, an essential component of tight junctions widely implicated in the barrier function of endothelium (19). The level of claudin-5 mRNA was significantly downregulated in L1-overexpressing luECs as compared with mock-transfected cells (Figure 4G), indicating that L1 negatively regulates its expression. The immunoblotting analysis also confirmed the decrease of claudin-5 in L1-transfected luECs (Supplemental Figure 10A). In line with these findings, the immunofluorescence (IF) staining of Panc02 tumors revealed that no or very little claudin-5 was present in $L 1^{\text {foxed }}$ tumor vessels, while the protein was readily detectable in the tumor vasculature of Tie2Cre; L1 1foxed mice (Supplemental Figure 10B), implicating L1 in the regulation of claudin- 5 expression in ECs.

Interendothelial junctions play a key role in the integrity of vascular barrier (20). Therefore, given the junctional alterations induced by L1, we tested whether endothelial permeability was modified by L1 overexpression. Indeed, the permeability of confluent luEC monolayers to FITC-labeled dextran was dramatically increased upon forced expression of L1 (Figure 4H), in agreement with the reduced permeability observed in the vasculature of Tie2Cre; $L 1^{\text {foxed }}$ mouse tumors (Figure 2, $\mathrm{G}$ and $\mathrm{H}$ ).

Taken together with our findings on L1-dependent alterations in tumor vessels (Figure 2), these data indicate that endothelial L1 
A

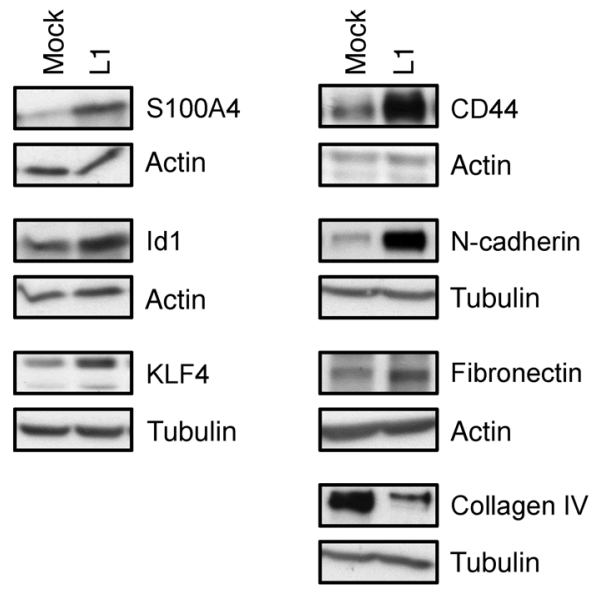

B

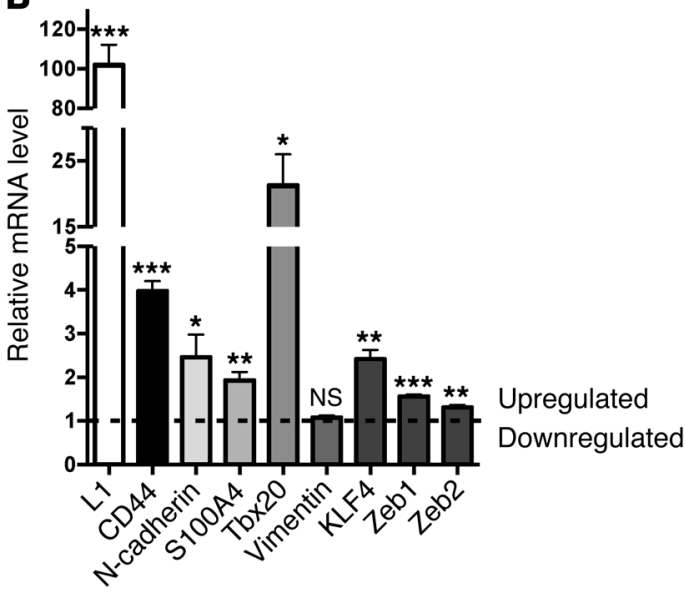

destabilizes the vasculature not only by altering pericyte coverage and collagen IV deposition, but also via EC-autonomous effects on the localization and/or expression of certain junctional components, therefore orchestrating intercellular adhesion.

$L 1$ promotes EndMT. The morphological and functional changes that L1 imparted on ECs, such as increased permeability, loosening of cell-cell junctions and a migratory phenotype, represent typical aspects of the EndMT, a process that has been causally linked to cancer progression (12). In the course of EndMT, ECs gain the expression of mesenchymal markers. Therefore, to verify whether L1 induced bona fide EndMT, we tested whether it promoted the acquisition of mesenchymal markers. The overexpression of L1 in luECs, indeed, resulted in the upregulation of S100A4, N-cadherin, fibronectin, and Id1 (Figure 5A), all events associated with EndMT (21). Notably, we also observed markedly reduced levels of collagen IV in L1-overexpressing luECs (Figure 5A), which, besides being another hallmark of EndMT (21), was consistent with the increased collagen IV deposition in L1-deficient Panc02 tumor vessels (Figure 2, E and F). Furthermore, L1-overexpressing luECs exhibited enhanced expression of the stemness-associated factors KLF4 and CD44 (Figure 5A), in line with the notion that EndMT is accompanied by the acquisition of a stem-like phenotype (21). Most of the markers above were validated by quantitative real-time PCR (qRT-PCR) (Figure 5B), implying a regulation at the transcriptional level. In contrast, the mesenchymal marker vimentin was not affected by L1 overexpression (Figure
Figure 5. L1 promotes EndMT. (A) Immunoblotting analysis of mock- and L1-transfected luECs for S100A4/FSP1, Id1, KLF4, CD44, N-cadherin, fibronectin, and collagen IV. Actin, tubulin, and vinculin served as loading controls. (B) qRT-PCR analysis of the indicated genes in mock- and L1-transfected cells. Transcript levels were normalized as described in Methods and are shown as fold changes in L1-transfected cells relative to mock-transfected cells $(n=3)$. ${ }^{*} P<0.05 ;{ }^{* *} P<0.01 ;{ }^{* *} P<0.001$.

5B). Besides mesenchymal markers, L1 was able to enhance the expression of transcription factors (TFs) that are thought to drive EndMT, such as Zeb1 and Zeb2, as well as Tbx20 (Figure $5 \mathrm{~B})$, which has been implicated in EndMT-like processes during heart development (22).

These data point to L1 as a regulator of EndMT, possibly implicating such an activity in L1-dependent alterations of EC behavior.

$L 1$ regulates EC transcriptome. In an attempt to elucidate the molecular mechanisms underlying the multiple roles of L1 in endothelium, we checked whether manipulating its expression affected the EC transcriptome. To this purpose, we compared the gene expression profiles of L1-overexpressing luECs with those of control luECs by Affymetrix microarray technology, and we used the Significance Analysis of Microarrays (SAM) (23) to identify genes whose expression was altered by L1. This analysis revealed a remarkable effect of $\mathrm{L} 1$ overexpression on luEC transcription (Figure 6A), with 361 genes that were upregulated and 580 that were downregulated ( $q$ value $<5 \%$; 1.5 -fold change difference; Supplemental Table 1). From the list of L1-regulated genes, we selected 16 candidates for qRT-PCR validation, based on their biological relevance. For all of them, including Cdk6, Adamts 9 , Hoxb9, Stmn2, Ebf1, Dll4, Vegfa, Vegfc, Ccnb1, Il13ra2 (Figure 6B), CD44, $\mathrm{N}$-cadherin, S100A4, Tbx20, Klf4 (Figure 5B), and Cldn5 (Figure $4 \mathrm{G}$ ), the regulation in L1-overexpressing luECs was confirmed by qRT-PCR. Many of these genes, including Stmn2, Ebf1, Dll4, Il13ra2, and Vegfc, exhibited a concordant L1 dependence upon siRNA-mediated silencing of L1 (Figure 6C), indicating that L1 is required and sufficient for their modulation.

Next, we used ingenuity pathway analysis (IPA) to analyze the effect of L1 overexpression on genes involved in biologically relevant functions. The "bio-functions" analysis revealed that L1 affects the expression profiles of several genes involved in celcycle regulation, DNA replication, cellular assembly, and organization (top 5 biofunctions, $P$ value $<10^{-7}$, Benjamini-Hochberg correction; Figure 6D), which is consistent with the L1-induced proliferation of ECs (Figure 4A). Importantly, IPA also identified genes involved in cell migration and development (Figure 6D). In particular, the gene expression profile of L1-transfected luECs was consistent with the activation of pathways involved in EC movement (Supplemental Figure 11), thus supporting our observations on L1-dependent functional changes (Figure 4, D and E).

L1 regulates EC function via the IL-6/JAK/STAT3 pathway. To get further insights about possible effectors of L1 biological function, we performed an unsupervised ingenuity upstream regulator analysis, which predicts the activation of specific gene expression modulators (i.e., TFs, microRNA, etc.). This analysis predicted the L1-induced activation of the TFs STAT1, STAT2, STAT3, IRF7, and ATF4. In particular, we identified a network of 105 L1-regulated genes downstream of the above-mentioned $5 \mathrm{TFs}$ (Figure 7A). 
A

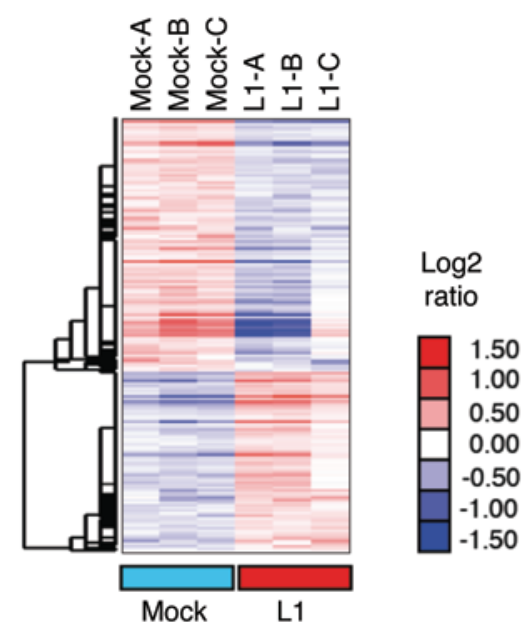

C

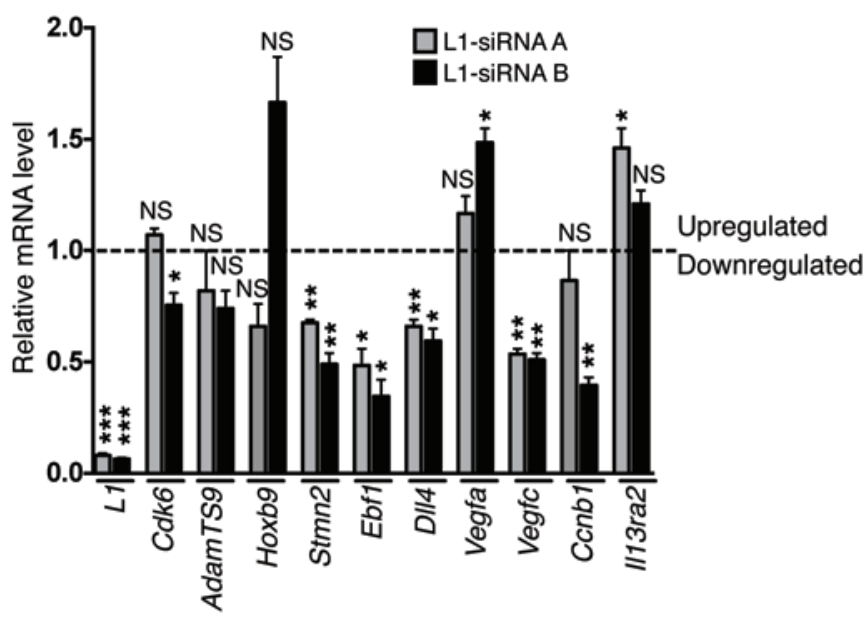

B

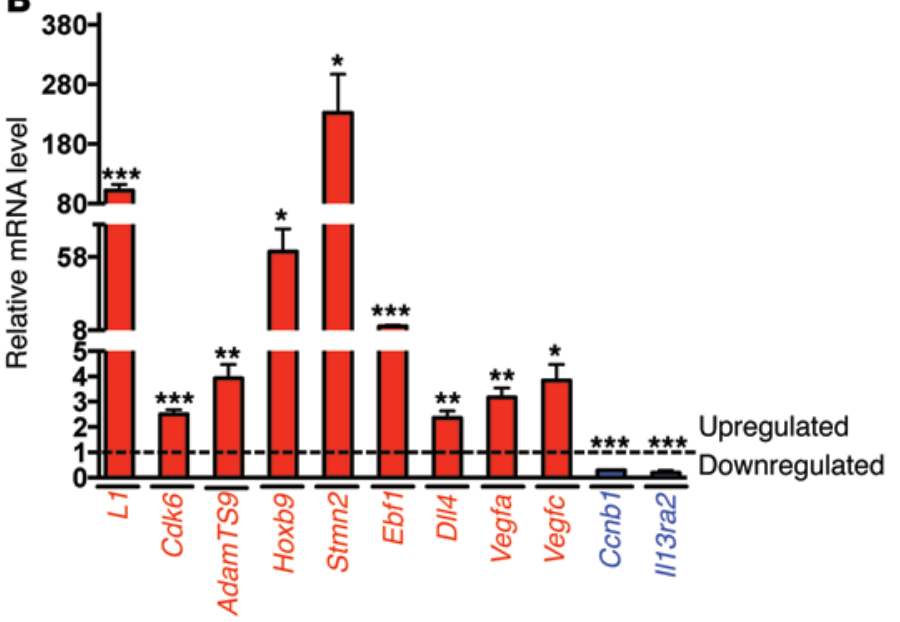

D L1-overexpression

- $\log (\mathrm{B}-\mathrm{H} P$ value $)$

$\begin{array}{lllllllllll}0 & 1 & 2 & 3 & 4 & 5 & 6 & 7 & 8 & 9 & 1011121314\end{array}$

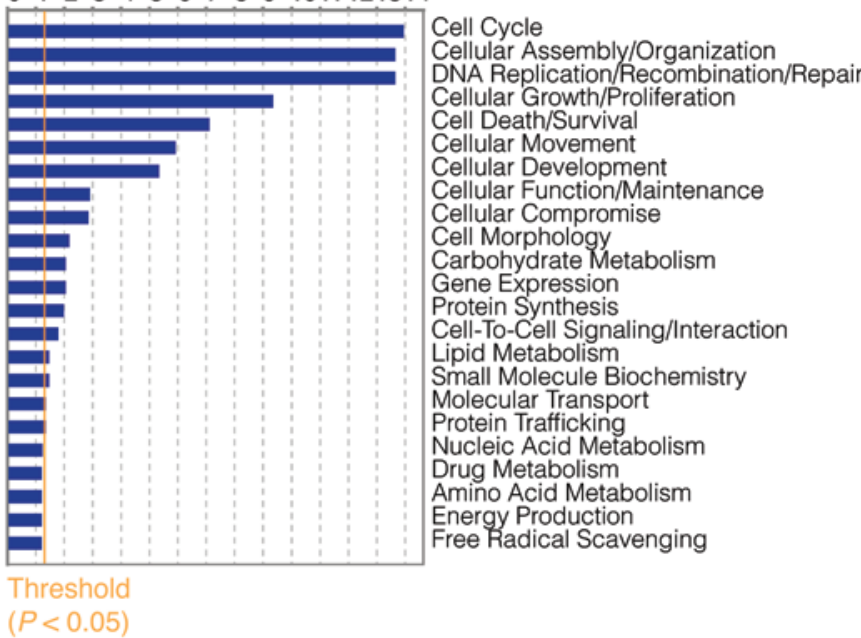

Figure 6. L1 regulates EC transcriptome. (A) Hierarchical clustering of genes differentially expressed in L1-transfected versus control luECs. Three independent experimental replicates of L1-overexpressing and control cells were screened by gene expression microarray. Data were log 2 transformed before clustering analysis. Red, upregulated genes; blue, downregulated genes. A total of 361 upregulated and 580 downregulated genes (i.e., 496 and 743 probe sets, respectively) were identified in L1-overexpressing cells. (B) qRT-PCR analysis of the indicated genes in mock- and L1-transfected luECs. Transcript levels were normalized as described in Methods and are shown as fold changes in L1-transfected cells relative to mock-transfected cells ( $n=3$ ). (C) qRT-PCR analysis of the indicated genes in luECs transfected either with 2 different L1 siRNAs ( $A$ and $B$ ) or with a control siRNA. Transcript levels are shown as fold changes in L1 siRNA-transfected cells relative to control cells $(n=3)$. ${ }^{*} P<0.05$; ${ }^{* *} P<0.01 ;{ }^{* *} P<0.001$. (D) IPA analysis of L1-regulated genes. The enriched biofunctions were selected based on their significance $(P<0.05$; Benjamini-Hochberg correction). Blue bars indicate the $-\log (P$ value $)$ of enrichment.

To investigate in more detail the molecular mechanisms underlying the effect of L1 on endothelium, we focused on STAT3, which has been implicated in tumor angiogenesis and EC activation (24). STAT3 activation is classically induced by IL-6 (25); therefore, we checked whether L1 affected the expression of this cytokine in luECs. Indeed, not only IL-6, but also its receptor IL-6R $\alpha$, was significantly upregulated in L1-overxpressing ECs, while the expression of the IL-6 coreceptor gp130 was not affected (Figure 7B). These observations were further confirmed by the increased levels of IL- 6 in the conditioned medium (Figure 7C) and of IL-6R $\alpha$ in the lysate of L1-overexpressing luECs (Figure 7D). The forced expression of L1 also resulted in the strong induction of STAT3 phosphorylation (Figure 7D), which was abolished by antibody-mediated neutralization of IL-6R $\alpha$ (Figure 7E). These results indicated that L1 promoted STAT3 activation via the IL-6/IL-6R $\alpha$ axis.
Next, we checked whether STAT signaling is involved in L1-dependent regulation of EC function. Since STAT activation occurs through JAK-mediated phosphorylation, we treated luECs with the JAK inhibitor I (JAKi) which, indeed, repressed L1-induced phosphorylation of STAT3 (Figure 7F). Importantly, JAK blockade repressed L1-dependent EC proliferation and migration (Figure 7, $\mathrm{G}$ and $\mathrm{H}$ ). Together, our transcriptomic, biochemical, and cell biological data support the notion that L1 regulates EC function via the JAK/STAT pathway.

Endothelial L1 is upregulated in human pancreatic carcinoma and in other tumor types. Based on our findings on the Panc02 orthotopic mouse model, we asked whether the expression of L1 in tumor vessels also occurs in human pancreatic carcinoma. Immunohistochemistry for L1 was performed on 18 tissue samples of PDAC and 11 samples of noncancerous pancreatic tissue, using 
A

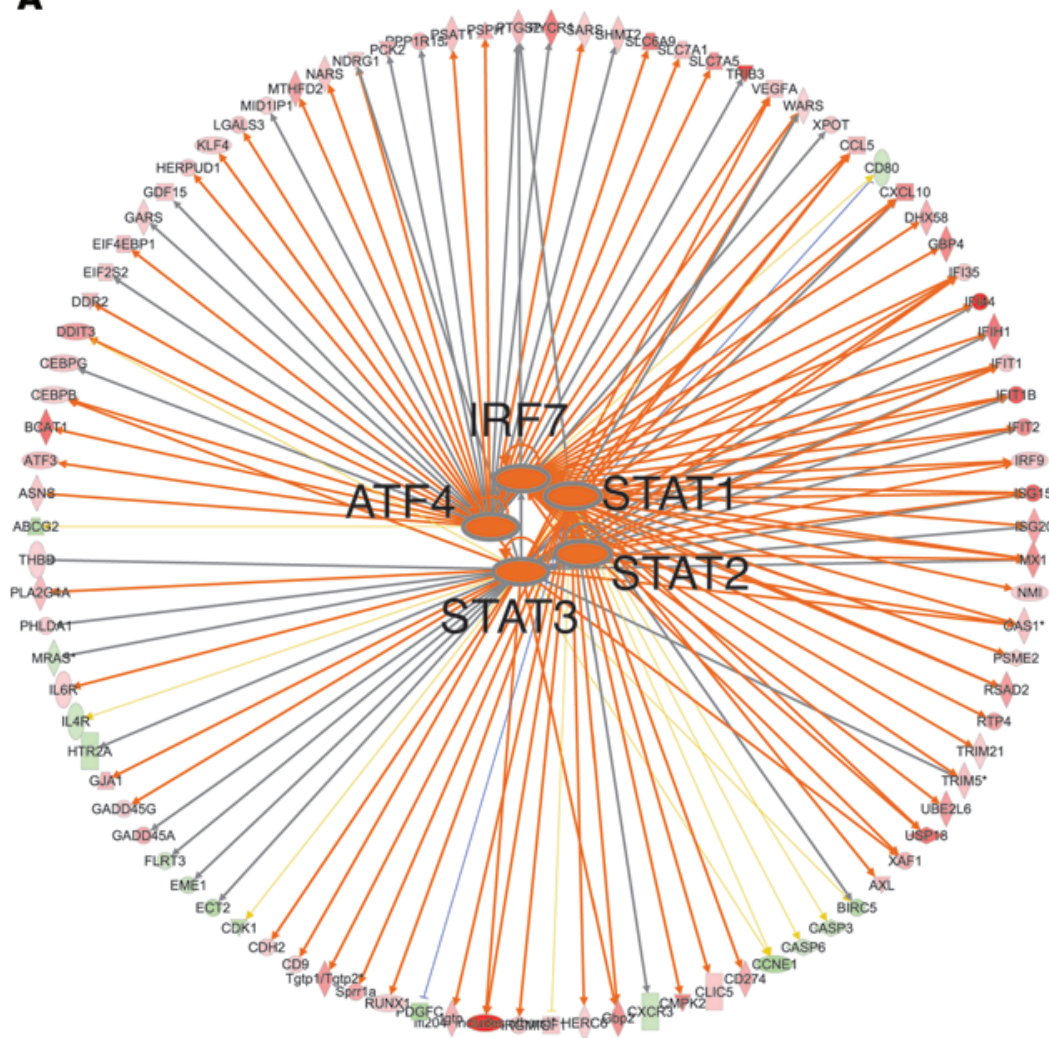

Consistent UP

D

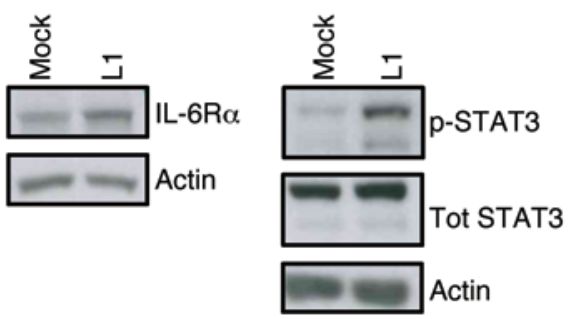

E Mock

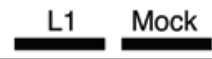

$\mathrm{L} 1$

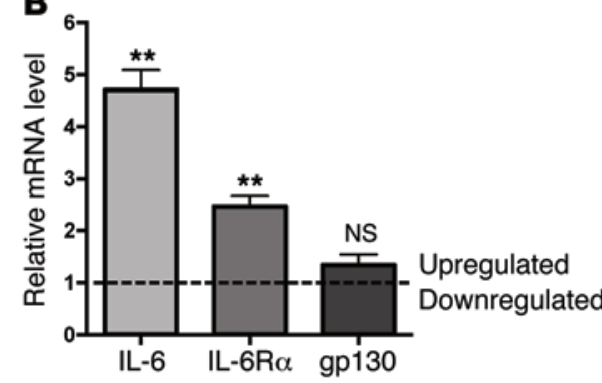

C
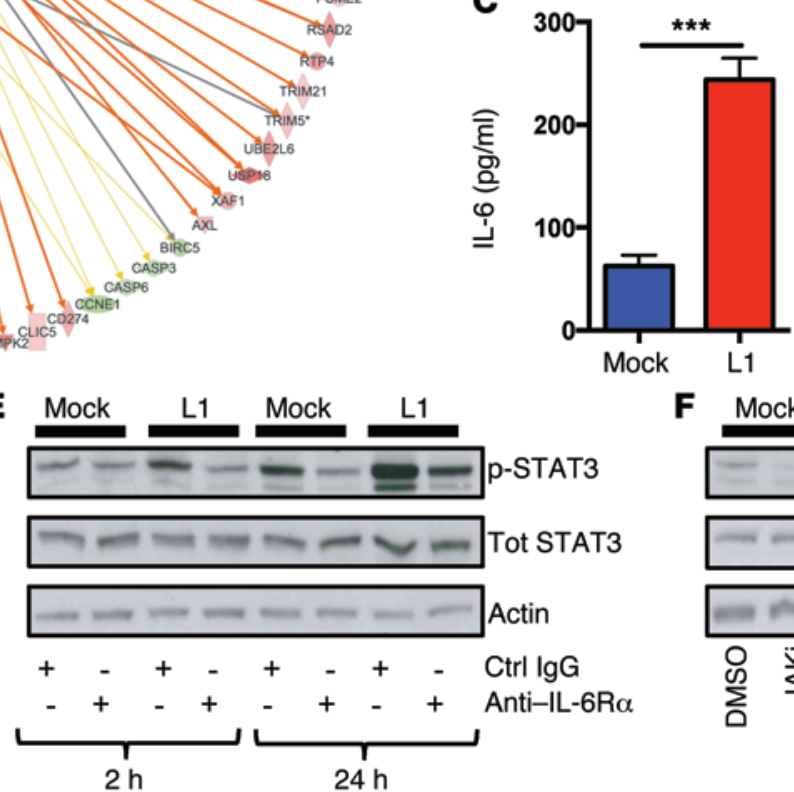

F Mock

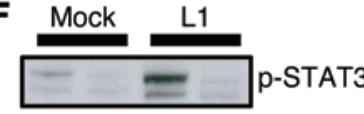

G

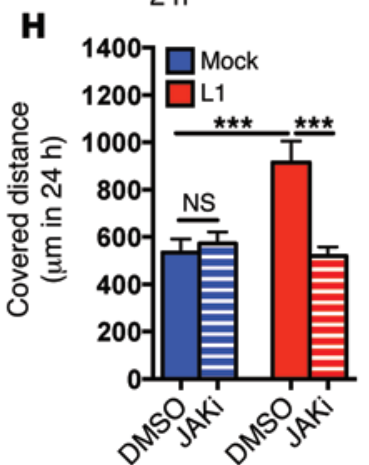

Figure 7. L1 regulates EC function via the IL-6/JAK/STAT3 pathway. (A) Gene network of L1-regulated genes. In bold, IPA-predicted upstream modulators. Lines connect modulators to direct targets, and colors indicate the consistency with the predicted activity with the expression change observed in L1-overexpressing luECs (i.e., target expression). Orange, consistent predicted activation of TFs; blue, consistent predicted inhibition of TFs; yellow, inconsistent predicted activation of TFs; grey, not defined activity. (B) qRT-PCR analysis of the indicated genes in mock- and L1-transfected luECs. Transcript levels were normalized as described in Methods and are shown as fold changes in L1-transfected cells relative to mock-transfected cells $(n=3)$. (C) The amount of IL-6 released in the culture medium by mock- and L1-transfected luECs was quantified by ELISA. (D) Immunoblotting analysis of mock- and L1-transfected luECs for IL-6R $\alpha$, phosphorylated STAT3, and total STAT3. (E) Immunoblotting analysis for phosphorylated and total STAT3 in mock- and L1-transfected luECs, treated either with anti-IL-6R $\alpha$ antibody or with control IgG. (F) Immunoblotting analysis for phosphorylated and total STAT3 in mock- and L1-transfected luECs, treated either with vehicle (DMSO) or with $20 \mu \mathrm{M}$ JAKi. Actin in D-F served as loading control. (C) Proliferation curves of mock- and L1-transfected luECs treated either with vehicle (DMSO) or with the indicated concentration of JAKi. (H) Mock- and L1-transfected luECs treated either with vehicle (DMSO) or with $20 \mu \mathrm{M}$ JAKi were subjected to 24 -hour migration assays. Data in $\mathbf{G}$ and $\mathbf{H}$ represent the mean \pm SD from a representative experiment performed in triplicate. ${ }^{* *} P<0.01 ;{ }^{* *} P<0.001$. 
A

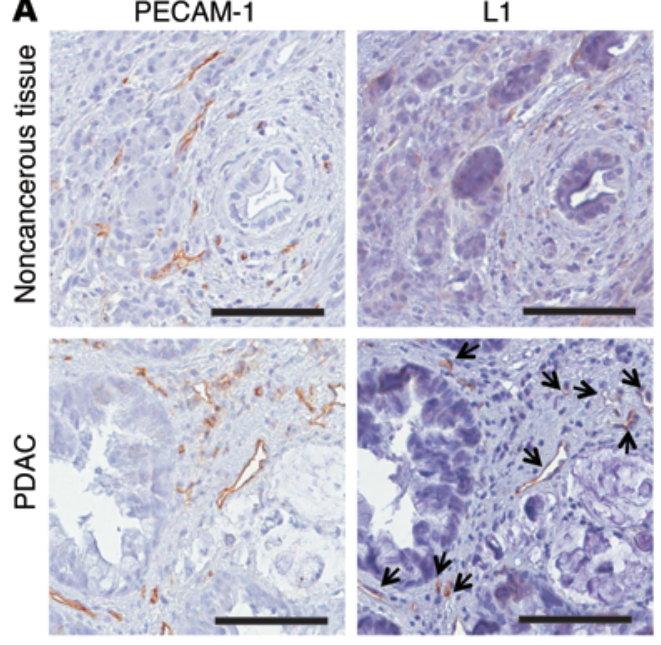

Figure 8. L1 is upregulated in human PDAC-associated vessels. (A) Consecutive sections from human noncancerous or PDAC tissue were stained for PECAM-1 (left panels) and L1 (right panels) in order to visualize L1-positive vessels (arrows). Scale bars: $100 \mu \mathrm{m}$. (B) Quantitation of L1-positive vessels in noncancerous $(n=11)$ and tumor tissue $(n=18)$, expressed as percentage of L1-positive over PECAM-1-positive vessels. ${ }^{* *} P<0.01$.
B

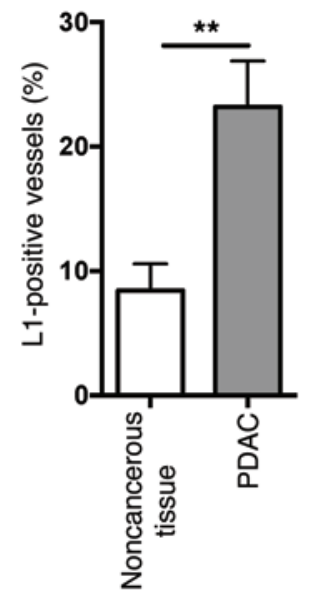

tumor vascularization and enhanced vessel normalization, thus delaying tumor growth. These findings were supported by in vitro data that revealed that L1 induces EC proliferation, migration, and tubulogenesis, and confers a mesenchymal phenotype to ECs.

Previous studies have shown that a soluble form of the L1's ectodomain enhances EC proliferation and migration (31-33), implying a model whereby exogenous (e.g., tumor cell derived) L1 stimulates ECs. While this remains a possibility, particularly in the case of L1-expressing tumors, our findings on the upregulation of L1 in cancer-associated vessels and on the proliferative and migratory response of L1-expressing ECs, point to a cell-autonomous effect of $\mathrm{L} 1$ in pathological vasculature.

It is noteworthy that $\mathrm{L} 1$ promotes the expression of various genes causally linked to tumor vascularization, such as VEGF-A, VEGF-C, Dll4, and HOXB9 (34-36). On the other hand, the endothelial expression of L1 itself is induced by classical angiogenic factors, including VEGF-A, ANGPTL4, TNF- $\alpha$, and IFN- $\gamma(10,11)$. Taken together, these observations an antibody against PECAM-1 to identify the vessels on consecutive sections. L1 expression was markedly enhanced in PDAC vasculature as compared with noncancerous tissue (Figure 8, A and B), confirming and extending previous observations (11). The presence of L1 in vascular endothelium was further validated by confocal microscopy on PDAC tissue costained for L1 and for the endothelial marker VE-cadherin (Supplemental Figure 12). Taken together with our data on the Panc02 tumor model and on cultured ECs, the expression pattern of L1 in clinical samples supports the hypothesis that vascular L1 contributes to pancreatic malignancy.

To test whether the vascular expression of L1 also occurs in other cancer types, we performed the immunohistochemical staining for L1 on tissue microarrays (TMAs) containing various tumors and their noncancerous tissue counterparts. As shown in Figure 9 and Supplemental Figure 13, in several tumor types, the percentage of L1-positive vessels was markedly higher as compared with their corresponding nonneoplastic tissues. Thus, the induction of $\mathrm{L} 1$ expression in the vasculature is common to a broad spectrum of human tumors.

\section{Discussion}

The expression of L1 in tumor vasculature has been reported in several cancer types, including breast, ovarian, colon, and pancreatic carcinoma $(10,11)$, neural tumors $(26)$, smooth muscle tumors (27), and melanoma (28). While vascular L1 has been implicated in the adhesion and transendothelial migration of L1-expressing cancer cells via homophilic binding $(11,29,30)$, it remains elusive whether L1 induces cell-autonomous effects in tumor endothelium and whether this has an effect on cancer development.

Here, we report for what we believe is the first time that L1 orchestrates the EC behavior in tumor vasculature. In particular, the endothelial deficiency of L1 in a mouse model of cancer led to enhanced vessel stability and decreased tumor angiogenesis, resulting in reduced tumor growth and metastasis and prolonged mouse survival. Consistently, antibody-mediated targeting of L1 reduced

implicate $\mathrm{L} 1$ as a central hub in the transmission and amplification of angiogenic stimuli within the tumor microenvironment.

Our data also indicate that L1 promotes vascular permeability. Since VEGF-A, ANGPTL4, TNF- $\alpha$ and IFN- $\gamma$ (i.e., the same cytokines that induce endothelial L1 expression) are among the most potent inducers of vascular permeability $(37,38)$, it is conceivable that L1 acts as a general effector of cytokine-induced vascular permeability.

Notably, we discovered that L1 overexpression induces EndMT, a process that may underlie, or at least contribute to, many aspects of the EC response to L1, such as migration and tubulogenesis as well as increased tumor angiogenesis and vascular permeability (12). Various lines of evidence support the notion that EndMT recapitulates most of the cellular and molecular events occurring during epithelial-to-mesenchymal transition (EMT) (12). The role of L1 in EMT has been clearly established in various experimental models (39-41), including tumor cells in which the expression of L1 per se promoted EMT $(42,43)$. We now provide evidence that $\mathrm{L} 1$ expression is sufficient to confer a mesenchymal phenotype to ECs, which entails not only an increased migratory activity but also the upregulation of several mesenchymal markers, such as N-cadherin, CD44, S100A4/FSP1, and fibronectin, and even of TFs that are considered to be EMT/EndMT drivers, namely KLF4, Zeb1, Zeb2, and Tbx2O (22, 44, 45). These findings suggest that L1 acts as a key mediator of the multiple EndMTinducing factors that can occur in tumor microenvironment and as a master orchestrator of cancer-associated EndMT (12).

One of the most intriguing and unexpected results of our study is the global effect of L1 on EC transcriptome, with the modulation of approximately 1,000 genes. While these include individual factors that per se might account for several aspects of the endothelial response evoked by L1, such as VEGF-A, VEGF-C, and Dll4 for tumor angiogenesis (see above), or occludin and claudin- 5 for vascular permeability (19), our data rather implicate L1 in the control of whole gene networks, resulting in the modulation of signaling pathways that mediate the EC response. 

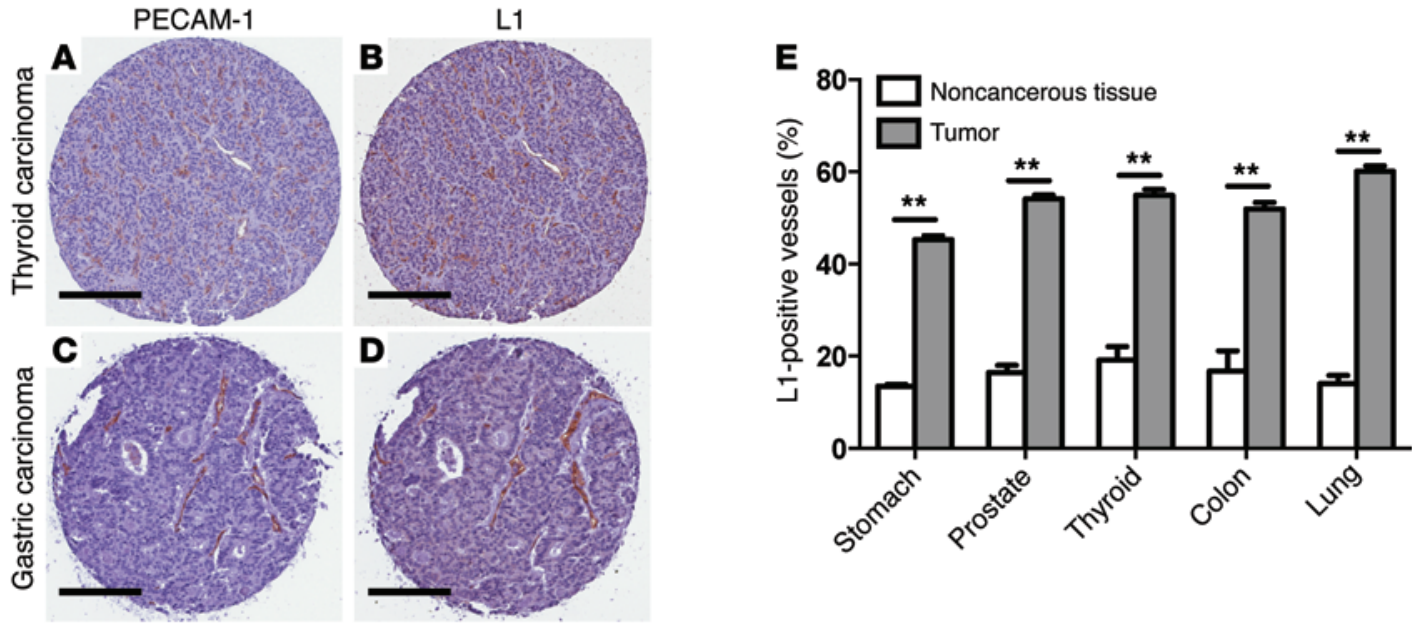

Figure 9. Vascular L1 is upregulated in various human tumor types. TMAs containing different tumor types and their normal tissue counterparts were stained for PECAM-1 (A and C) and L1 (B and D). Examples of thyroid carcinoma (A and B) and gastric carcinoma (C and $\mathbf{D})$ are shown. Scale bars: $200 \mu \mathrm{m}$. (E) Quantitation of L1-positive vessels in noncancerous and tumor tissue expressed as percentage of L1-positive over PECAM-1-positive vessels. ${ }^{* *} P<0.002$ ( $n=5$ for each tissue type).

In this context, it is noteworthy that L1 induces the expression of IL- 6 and IL-6R $\alpha$. IL- 6 is a potent angiogenic cytokine that promotes neovascularization in various solid tumors, and it has been proposed not only as a therapeutic target for antiangiogenic therapies but also as a biomarker to predict the response to such treatments (46). Thus, it is reasonable to speculate that the IL-6/ IL6-R $\alpha$ axis acts as a key mediator of the angiogenic response elicited by vascular L1, an intriguing hypothesis that warrants further investigation. In addition, L1-mediated regulation of IL-6 expression might also occur in different biological contexts where IL-6 signalling has been implicated. For example, the IL-6/IL-6R $\alpha$ system plays a major role in inflammation and in several cancer cell functions $(47,48)$, and our findings imply that the established function of L1 in both pathological conditions $(8,10)$ might be accounted for, at least to some extent, by the induction of IL-6 expression and activity.

Among the signalling cascades that are elicited by IL-6, the JAK/STAT pathway appears particularly important in the context of L1-dependent regulation of EC function. Indeed, L1 induces high phosphorylation of STAT3, a TF that plays a key role in EC activation and pathological angiogenesis $(49,50)$, consistent with the hypothesis that L1-dependent regulation of tumor vasculature is mediated by the IL-6/JAK/STAT pathway. Our data on the inhibition of L1-induced EC proliferation and migration upon blockade of JAK/STAT signaling not only support this hypothesis, but provide mechanistic insights into the role of L1 in pathological vessels and shed light on a signaling axis that links L1 to the JAK/ STAT pathway. Given the broad spectrum of functions that have been ascribed to L1 in different cellular contexts, including cellcell adhesion, axon guidance, tumor cell invasion, and stem cell self renewal $(7,8,51)$, we propose that at least some of these activities are mediated by JAK/STAT signaling.

The expression and function of L1 in cancer vessels has relevant translational implications. First, it provides the rationale to test L1 as an imaging biomarker suitable for visualizing pathological angiogenesis. In this application, due to its absence or low levels in normal vasculature, L1 might show higher specificity than other molecular biomarkers that are currently in use or under clinical testing, such as integrins and VEGFR2 (52). The feasibility of this approach is supported both by the preclinical imaging of cancer vessels by targeting other immunoglobulinlike adhesion molecules such as NCAM (53) and by the suitability of L1 antibodies for tumor-imaging purposes as shown in mouse models (54). Second, based on the positive role of L1 in tumor neovascularization, in EC proliferation and migration, and in EndMT, it is reasonable to speculate that neutralizing L1 could represent a novel antiangiogenic strategy. Indeed, we showed that treating tumor-bearing mice with an L1-neutralizing polyclonal antibody delays tumor growth and reduces tumor vascularization, strengthening the rationale for developing L1-targeting agents as therapeutic tools. In this context, promising results have been obtained with anti-L1 monoclonal antibodies in preclinical models of solid tumors and of endometriosis $(55,56)$. Third, the observation that L1 destabilizes cancer-associated vessels implies that targeting endothelial L1 and interfering with its function might result in vessel normalization, a process that has been proposed to improve the delivery into the tumor of systemically administered chemotherapeutics (4). Thus, our results set the stage for exploring the clinical relevance of L1 expression and function in cancer vessels, possibly opening new avenues for targeted treatments of malignancies.

\section{Methods}

\section{Mice}

Tie2-Cre; $L 1^{\text {floxed }}$ mice were generated in the C57BL/6 genetic background as previously described (10). Since the L1 gene is located on chromosome $\mathrm{X}$ (and hence, only 1 copy is present in the male genome), Cre-mediated ablation of $L 1$ was expected to be more efficient in males. Therefore, only Tie2-Cre-positive males carrying the floxed L1 allele were used throughout the study, with $L 1^{\text {foxed }}$ males serving as controls. 


\section{In vivo models}

Pancreatic carcinoma model. The syngeneic mouse model of pancreatic cancer has been described previously (13). Briefly, 10- to 12-week-old C57BL/6 mouse males were anesthetized by intraperitoneal injection of $500 \mathrm{mg} / \mathrm{kg}$ avertin (Sigma-Aldrich), the stomach was exteriorized via abdominal midline incision, and Panc02 tumor cells $\left(1 \times 10^{6}\right.$ cells in $30 \mu \mathrm{l}$ PBS) were injected into the head of the pancreas using a 29-gauge needle. The intrapancreatic injection was considered successful with the appearance of a fluid bleb without intraperitoneal leakage. Peritoneum and abdominal wall were closed with individual surgical sutures. Where indicated, mice were treated every 48 hours with $7 \mathrm{mg} / \mathrm{kg}$ of affinity-purified anti-L1 polyclonal antibodies (obtained from rabbits immunized with mouse L1-Fc) or control, nonimmune rabbit IgG (Sigma-Aldrich), starting from the day after Panc02 injection. At day 14, primary tumors were removed, and tumor weight and volume were recorded. Tumor volume was calculated using the formula $V=\pi \times(d 1 \times d 2 \times d 3) / 6$, where $d 1, d 2$, and $d 3$ are the 3 tumor axes. To assess tumor invasion to adjacent organs and metastasis, mice were sacrificed at day 26 and subjected to whole-mount body fixation with $4 \%$ paraformaldehyde. Necropsy was performed by the Mouse and Animal Pathology Laboratory, Fondazione Filarete (Milan, Italy).

Hemangioma model. For EC transplantation, $1 \times 10^{5}$ mock- or L1transfected mouse luECs in $200 \mu \mathrm{l}$ of PBS was injected subcutaneously into the right flank of CD-1 nude (nu/nu) mice (9 weeks old, female; Charles River Laboratories) as previously described (18). Hemangioma volumes were recorded at the indicated time points and calculated using the formula $V=\pi \times\left(d^{2} \times D\right) / 6$, where $d$ and $D$ are the minor and the major hemangioma axes, respectively.

\section{Chemicals and antibodies}

The JAK inhibitor 1 was provided by Calbiochem. The following monoclonal antibodies were used as indicated: anti-mouse L1 (clone S10.33 [ref. 10, $5 \mu \mathrm{g} / \mathrm{ml}$ in IF]; clone 555 [ $1 \mu \mathrm{g} / \mathrm{ml}$ in IF]; clone 324 [ref. 6] hybridoma supernatant [1:2 in IF]; and clone I4.2 [ref. 10] hybridoma supernatant [1:2 in Western blotting (WB)]); anti-human L1 (clone UJ127; 1:30 in IHC on human tissues; Thermo Scientific); anti-PECAM-1 (clone 2H8; 1:500 in IF on cells and mouse tissues, Millipore; clone JC70A; 1:30 in IHC on human tissues, Dako); anti-claudin-5 (clone 4C3C2; 1:200 in IF, 1:250 in WB, Life Technologies); anti-actin (clone AC-40; 1:1000 in WB; Sigma-Aldrich); anti- $\alpha$-catenin (clone $5 / \alpha$-catenin; 1:1000 in WB; BD Biosciences); anti- $\beta$-catenin (clone $14 / \beta$-catenin; $1: 200$ in IF and 1:500 in WB; BD Biosciences); anti- $\gamma$-catenin (clone $15 / \gamma$-catenin; $1: 1000$ in $\mathrm{WB}$; BD Biosciences); anti-CD44 (clone IM7; 1:100 in WB; BD Biosciences); anti-JAM-A (clone BV19/BV20) (57); anti-N-cadherin (clone 32/N-cadherin; 1:2000 in WB; BD Biosciences), anti-p120-catenin (clone 98/pp120; 1:1000 in WB; BD Biosciences); anti-phosphoStat3 (Cell Signaling, clone D3A7; 1:1000 in WB); anti-Stat3 (clone 124H6; 1:1000 in WB; Cell Signaling); anti-VE-cadherin (clone 11D1.4; 1:200 in IF on cells and mouse tissues; BD Biosciences); antivinculin (clone hVIN-1; 1:2000 in WB; Sigma-Aldrich); anti-tubulin (clone DM1A; 1:2000 in WB; Sigma-Aldrich). The following polyclonal antibodies were used as indicated: anti-collagen IV (1:900 in IF and 1:400 in WB; Serotec); anti-fibrin/fibrinogen (1:1000 in IF; Dako); anti-fibronectin (1:4000 in WB; Abcam); anti-S100A4/FSP1 (1:1000 in WB; Millipore); anti-Id1 (C-20; 1:400 in WB; Santa Cruz Biotechnology Inc.); anti-IL-6R $\alpha$ (1:1000 in WB; R\&D Systems);
anti-Ki-67 (1:100 in IF; Abcam); anti-KLF4 (1:1000 in WB; R\&D Systems); anti-mouse L1 (obtained by rabbit immunization with mouse L1-Fc; $1 \mu \mathrm{g} / \mathrm{ml}$ in IF and WB); anti-PECAM-1 (M-20; 1:500 in WB; Santa Cruz Biotechnology Inc.); anti-NG-2 (1:200 in IF; Millipore); anti-phospho-histone H3 (1:250 in IF; Millipore); anti-podocalixin (1:400 in IF; R\&D Systems); anti-VE-cadherin (C-19; 1:500 in WB and 1:200 in IF on human tissues; Santa Cruz Biotechnology Inc.); anti- $\beta$-tubulin III (1:500 in IF; Covance). Antibodies used for FACS analysis are listed in the corresponding section.

\section{Cells}

Mouse luECs were immortalized with polyoma middle $\mathrm{T}$ antigen as previously described (18) and cultured in MCDB131 medium (Gibco; Life Technologies) supplemented with 20\% FBS (Invitrogen), 2 mM L-glutamine (Lonza), $1 \mathrm{mM}$ Na-pyruvate (Gibco; Life Technologies), $100 \mu \mathrm{g} / \mathrm{ml}$ heparin (Sigma-Aldrich), and $50 \mu \mathrm{g} / \mathrm{ml} \mathrm{EC} \mathrm{growth} \mathrm{supple-}$ ment (ECGS) obtained from calf brain. ECs were seeded on $0.1 \%$ gelatin (Sigma-Aldrich), except as otherwise indicated. To enhance EC adhesion, plates were coated with glutaraldehyde-crosslinked gelatin as follows. Plates were incubated overnight with $1 \%$ gelatin at $37^{\circ} \mathrm{C}$ followed by a crosslinking with $2 \%$ glutaraldehyde for 15 minutes at room temperature (RT). Glutaraldehyde was replaced with $70 \%$ ethanol for 1 hour at RT. After 5 washes with PBS, plates were incubated for 2 hours at $37^{\circ} \mathrm{C}$ with $2 \mathrm{mM}$ glycine in PBS. Prior to cell seeding, plates were washed 5 times with PBS.

Murine Panc02 (pancreatic carcinoma) and MOVCAR7 (ovarian carcinoma) cell lines were provided by S. Sebens (Institute for Experimental Medicine, Kiel, Germany) and D. Connolly (Fox Chase Cancer Center, Philadelphia, Pennsylvania, USA), respectively, and cultured in RPMI 1640 medium (Lonza) supplemented with 10\% FBS, 2 mM L-Glutamine, and $1 \mathrm{mM}$ Na-pyruvate. All cells were cultured in a humidified incubator with $5 \% \mathrm{CO}_{2}$.

\section{Cell transfection and RNA interference}

Mouse cDNA encoding full-length L1 was cloned into the pcDNA3.1/ Hygro(-) expression vector (Invitrogen) using standard DNA cloning procedures. The parental pcDNA3.1/Hygro vector and pcDNA3.1/ Hygro-L1 were amplified in competent bacteria cells (TOP10) and purified using Maxi-Prep kits (QIAGEN) according to the manufacturer's instructions. Transfection with either pcDNA3.1/Hygro or pcDNA3.1/Hygro-L1 was performed with LipofectAMINE 2000 (Invitrogen) according to the manufacturer's instructions. Transfected cells were selected with $300 \mu \mathrm{g} / \mathrm{ml}$ of Hygromycin-B (Roche) and maintained under selection conditions as a bulk cell population.

Stealth RNAi Duplexes and the corresponding Medium GC Stealth RNAi Control Duplexes (Invitrogen) were used to knock down L1 in murine ECs. The following L1 target sequences were used: 5 '-CCUGGUACCCGGACCAUCAUUCAAA-3' (siRNA A) and 5'-UGCACCUUUCUUCUCAAUUGCGCUC-3' (siRNA B). ECs were subjected to 2 rounds of transfection with $40 \mathrm{nM}$ siRNA the 2 days prior to the experiment. Transfection was performed with LipofectAMINE 2000 (Invitrogen) according to the manufacturer's instructions.

\section{Cell proliferation assay}

ECs were seeded at a density of $2 \times 10^{3}$ cells/well in 96-well plates coated with glutaraldehyde-crosslinked gelatin. After overnight incubation in medium containing $5 \% \mathrm{FBS}$, cells were stimulated with 
medium containing 20\% FBS, $100 \mu \mathrm{g} / \mathrm{ml}$ heparin, and $50 \mu \mathrm{g} / \mathrm{ml}$ ECGS. Where indicated, cells were treated with JAK inhibitor 1 or vehicle (DMSO) or with $3 \mu \mathrm{g} / \mathrm{ml}$ of polyclonal anti-L1 antibodies or control, nonimmune rabbit IgG throughout the assay. Cells were fixed at 0, 24, 48 , and 72 hours, followed by staining with $0.1 \%$ crystal violet in $20 \%$ methanol. Bound dye was solubilized with $10 \%$ acetic acid, and the absorbance at $590 \mathrm{~nm}$ was measured. Cell growth was normalized on absorbance measured at 0 hours. The experiments were performed in quintuplicate and repeated at least 3 times.

\section{Cell migration assay}

To assess cell migration, we employed the wound-healing assay. Briefly, confluent monolayers of luECs seeded on fibronectin-coated $\left(1 \mu \mathrm{g} / \mathrm{cm}^{2}\right) 24$-well plates were starved for 24 hours in medium containing $0.5 \%$ FBS medium. Monolayers were wounded with a plastic pipette tip to induce EC migration into the wound, and images were acquired at 0 and 24 hours. Where indicated, cells were pretreated for 1 hour with $20 \mu \mathrm{M}$ JAK inhibitor 1 or vehicle (DMSO), or with $3 \mu \mathrm{g} / \mathrm{ml}$ anti-L1 polyclonal antibodies or control, nonimmune rabbit IgG; such treatments were maintained throughout the assay. Optical images of the wounds were acquired at 0 and 24 hours. The width of the wounds was measured with ImageJ (http://imagej.nih.gov/ij/) software, and the distance covered luECs, expressed as $\mu \mathrm{m}$ in 24 hours, was calculated according to this formula: (wound width at $\mathrm{O}$ - wound width at 24 hours) $/ 2$. The experiments were repeated 3 times, each time with triplicate wells.

\section{Tube formation assay}

A Matrigel-based tubulogenesis assay was performed to assess the ability of ECs to form an organized capillary-like network. Confluent ECs were starved overnight with medium containing 1\% FBS. Growth factor-reduced Matrigel (BD Biosciences) was thawed overnight at $4^{\circ} \mathrm{C}$ on ice and, the day of the assay, plated on the bottom of a 96 -well plate and left at $37^{\circ} \mathrm{C}$ for 1 hour for gelification. Thereafter, $1 \times 10^{4}$ cells/well were seeded on Matrigel and incubated at $37^{\circ} \mathrm{C}$. Where indicated, cells were treated with $3 \mu \mathrm{g} / \mathrm{ml}$ anti-L1 polyclonal antibodies or control, nonimmune rabbit IgG. Optical images of the wells were acquired after 8 hours at $\times 4$ magnification. The tubes in each well were manually counted. The experiments were repeated 3 times, each time with triplicate wells.

\section{Vascular permeability assays}

In vitro. The luECs were cultured on Transwell inserts (clear, 24-well plate, $0.4-\mu \mathrm{m}$ pores; $6.5 \mathrm{~mm}$ diameter; tissue culture-treated; Costar), coated with glutaraldehyde-crosslinked gelatin, and allowed to form confluent monolayers (typically after 4 days). The day of the experiment, the medium in the upper chamber was replaced with complete medium containing $1 \mathrm{mg} / \mathrm{ml}$ FITC-labeled 40-kDa dextran (Sigma-Aldrich). At each time point, $50 \mu \mathrm{l}$ of medium in the bottom chamber were taken, and fluorescence was measured at $488 \mathrm{~nm}$. The medium taken from the bottom chamber was replaced each time with fresh complete medium in order to maintain a constant volume in the bottom chamber. The experiments were performed in quintuplicate and repeated 3 times.

In vivo. Texas red-labeled 40-kDa dextran (Life Technologies) was administered intravenously by retroorbital injection into anesthetized Panc02 tumor-bearing mice $(0.25 \mathrm{mg} /$ mouse). Thirty minutes after injection, mice were sacrificed and their tumors were fixed in $2 \%$ para- formaldehyde and embedded in paraffin. Sections were then subjected to IF costaining with rat anti-CD34 (BD; clone RAM34; 1:100) and rabbit anti-Texas red antibodies (1:100; Life Technologies). Vascular permeability was assessed as the ratio between the number of leaky vessels (i.e., vessels showing perivascular, extravasated Texas red staining) and the total number of CD34-positive vessels in 5 randomly selected fields (13).

\section{Gene expression profiling}

ECs were seeded on plates coated with glutaraldehyde-crosslinked gelatin and cultured in complete medium for 4 days to reach confluence. Total RNA was extracted with RNeasy Mini Kit (QIAGEN). Quality control of the RNA samples was performed using Agilent Bioanalyzer 2100 (Agilent Technologies). Three different RNA extractions were processed for each of the cell lines under analysis. Each sample was labeled and hybridized to a Mouse Gene 1.0 ST Genechip array according to the manufacturer's instructions (Affymetrix). Data were normalized using the Robust Multi-array Average (RMA) (ref. 58; raw and normalized data were deposited in the NCBI's Gene Expression Omnibus [GEO GSE45859]).

All analyses were performed on $\log _{2}$ data using parametric tests. BRB ArrayTools (http://linus.nci.nih.gov/BRB-ArrayTools.html) was used to run SAM analysis (http://www-stat.stanford.edu/ tibs/SAM/). A false discovery rate of less then $5 \%$ (i.e., the $q$ value) was used to select statistically significant differentially expressed genes. Cluster 3.0 for Mac OS X (http://bonsai.hgc.jp/ mdehoon/software/cluster/) and Java Treview (http://jtreeview.sourceforge.net) were used for hierarchical clustering analysis using the uncentered correlation metric and centroid clustering method.

Ingenuity Pathway Analysis and Upstream Regulator Analysis were performed using the online available web tool (http://www. ingenuity.com/). For gene network analysis, only direct relationships in mammals (i.e., human, mouse, and rat) were considered. $P$ values for biofunction enrichment were corrected for multiple testing using the Benjamini-Hochberg correction.

\section{Immunoblotting}

ECs were cultured on petri dishes coated with glutaraldehyde-crosslinked gelatin for 4 days. Where indicated, cells were incubated for 2 or 24 hours with $2 \mu \mathrm{g} / \mathrm{ml}$ goat polyclonal anti-IL-6 R (R\&D Systems) or control goat IgG. Total proteins were extracted by solubilizing cells in boiling Laemmli buffer (4\% SDS, 16\% glycerol, $40 \mathrm{mM}$ Tris- $\mathrm{HCl}[\mathrm{pH}$ 6.8]). Lysates were incubated for 15 minutes at $90^{\circ} \mathrm{C}$ to allow protein denaturation and then centrifuged for 5 minutes at $14,000 \mathrm{~g}$ to discard cell debris. The supernatants were collected, and the concentration of protein was determined using a BCA Protein Assay Kit (Pierce) according to the manufacturer's instructions. Equal amounts of protein were separated on SDS polyacrylamide gel (SDS-PAGE), transferred to a Protran Nitrocellulose Transfer Membrane (Whatman), and blocked for 1 hour at RT in TBS, 0.1\% Tween 20 containing 5\% nonfat milk or BSA (blocking solution). The membranes were incubated overnight at $4^{\circ} \mathrm{C}$ or for 1 hour at RT with primary antibodies diluted in blocking solution. Membranes were then incubated with horseradish peroxidase-conjugated secondary antibodies (Bio-Rad). The signal was detected by the ECL system (GE Healthcare) using Hyperfilm (Amersham Biosciences). The molecular masses of proteins were estimated relative to the electrophoretic mobility of contransferred prestained protein marker Precision Plus Protein Standards (Bio-Rad). 


\section{qRT-PCR analysis}

ECs were cultured on petri dishes coated with glutaraldehyde-crosslinked gelatin for 4 days. Total RNA was isolated by extraction with RNeasy Mini Kit (QIAGEN), and $1 \mu \mathrm{g}$ was reverse-transcribed with random hexamers (SuperScript Vilo cDNA Synthesis Kit; Invitrogen) according to the manufacturer's instructions. cDNA (5 ng) was amplified in triplicate in a reaction volume of $15 \mu$ l with the TaqMan Gene Expression Assay (Applied Biosystems) and an ABI/Prism 7900 HT thermocycler (Applied Biosystems) using a pre-PCR step of 10 minutes at $95^{\circ} \mathrm{C}$, followed by 40 cycles of 15 seconds at $5^{\circ} \mathrm{C}$ and 60 seconds at $60^{\circ} \mathrm{C}$. Preparations of RNA template without reverse transcriptase were used as negative controls. For each sample, the expression level was normalized against the geometric mean of the housekeeping genes encoding GAPDH and 18S. Normalized expression changes were determined with the comparative threshold cycle (Ct) method (59).

\section{IF}

ECs were cultured on 35-mm petri dishes coated with glutaraldehydecrosslinked gelatin for 4 days and then fixed with $4 \%$ PFA for 10 minutes at room temperature. Panc02 tumors were fixed with $2 \%$ PFA overnight at $+4^{\circ} \mathrm{C}$ and embedded in OCT after a graded sucrose series. Fixed cells and tumor sections $(5 \mu \mathrm{m})$ were permeabilized with PBS and $0.5 \%$ Triton $\mathrm{X}-100$ and incubated for 1 hour at RT with a blocking solution of PBS, $2 \%$ BSA, $5 \%$ donkey serum, and 0.05\% Triton X-100. Samples were then incubated overnight at $+4^{\circ} \mathrm{C}$ with primary antibodies diluted in blocking buffer, followed by secondary antibodies ( 2 hours at room temperature). Samples were then washed and mounted with Vectashield mounting medium with DAPI (Vector Laboratories). For staining of L1 on cells or on tumor sections, the permeabilization step was omitted and Tween 20 was used instead of Triton X-100 in all the buffers. TUNEL staining (Roche) was performed according to the manufacturer's instructions.

Confocal microscopy was performed with a Leica SP2 confocal microscope equipped with a motorized stage and violet (405 $\mathrm{nm}$ laser diode), blue (488 nm Argon), yellow (561 nm laser diode), and red (633 $\mathrm{nm}$ HeNe laser) excitation laser lines. In some cases, tile scans of the tumor sections were acquired using $\times 20$ magnification. Mosaic images were created using the Leica LCS software.

The number of $\mathrm{Ki}-67^{+}, \mathrm{PHH}^{+}$, or TUNEL ${ }^{+}$cells $/ \mathrm{mm}^{2}$ were calculated by counting the number of positive cells in 10 different fields at $\times 63$ magnification for each section. Density of PECAM-1-positive blood vessels was measured on images of tumor sections acquired as reported above and quantified with a custom-written plug-in of ImageJ software.

Vessel pericyte coverage was calculated on the images of tumor sections and quantified with a custom-written plug-in, as previously described (60). Collagen IV and fibrin(ogen) deposition was measured on images of tumor sections and quantified with Image J as the mean of the fluorescence intensity of the pixels in the tumor area analyzed. Fluorescence microscopy was performed with a Olympus BX61 automated upright wide-field microscope equipped with a Photometrics Coolsnap Camera.

\section{IL-6 detection}

ECs were seeded on plates coated with $0.1 \%$ gelatin and cultured in complete medium for 4 days to reach confluence. At day 4 , medium was replaced by fresh complete medium, and supernatants were collected after 24 hours. Mouse IL- 6 was quantitated using the Quantikine ELISA Kit (R\&D Systems) according to the manufacturer's instructions.

\section{Immunohistochemistry}

The clinicopathological data of PDAC patients are described in Supplemental Table 2. Formalin-fixed, paraffin-embedded specimens were prepared from both cancerous $(n=18)$ and noncancerous pancreatic tissue samples $(n=11)$. Sections were rehydrated through xylene and graded alcohols. Antigen retrieval was accomplished using $1 \mathrm{mM}$ EDTA and $0.05 \%$ Tween. Samples were incubated with $3 \% \mathrm{H}_{2} \mathrm{O}_{2}$ for 5 minutes, followed by blocking in $2 \%$ goat serum in PBS for 1 hour. Primary antibodies were incubated for 2 hours at RT in 2\% goat serum. Horseradish peroxidase-conjugated secondary antibodies were used. Samples were developed with DAB and counterstained with hematoxylin.

The vascular expression of $\mathrm{L} 1$ on tissue sections was measured as the ratio between L1-positive vessels and the total number of vessels identified by PECAM-1 staining on consecutive sections. The analysis was performed on 10 fields per sample at $\times 40$ magnification.

TMAs were prepared with formalin-fixed, paraffin-embedded samples from different tumor types (thyroid, gastric, colon, lung, and prostate carcinoma) and from their normal tissue counterparts. Representative areas were first selected on H\&E-stained sections by a trained pathologist; 2 representative core biopsies for every tissue block were included in the TMAs. TMAs were assembled on a custombuilt tissue arrayer (Beecher Instruments) as previously described (61). TMAs were stained for PECAM-1 and L1, and the vascular expression of L1 was measured on serial sections as described above.

\section{FACS analysis}

Tumors were collected 14 days after Panc02 injection, washed in PBS, and digested in RPMI medium containing 0.1\% collagenase type I (Gibco; Life Technologies) and $10 \mu \mathrm{g} / \mathrm{ml}$ DNase (Roche) for $45 \mathrm{~min}$ utes at $37^{\circ} \mathrm{C}$. Digested tissue was passed 10 times through a 20 -gauge needle and filtered through a $40-\mu \mathrm{m}$ pore-sized mesh. After red blood cell lysis, cells were resuspended in FACS buffer (PBS, 1\% FBS, 0.05\% $\mathrm{NaN}_{3}$ ) and subjected to FACS staining. After a blocking incubation in FACS buffer containing 1\% normal mouse serum (Sigma-Aldrich) for 45 minutes on ice, cells were stained for 45 minutes on ice with the indicated antibodies against different immune cell markers. Finally, cells were washed 3 times and fixed with 1\% PFA. Percentage of positive cells was measured by FACSCalibur flow cytometer and analyzed with CellQuest software.

The following monoclonal antibodies, all purchased by BD Biosciences except otherwise indicated, were used for FACS staining: PE anti-CD4 (clone RM4-5; 1:200), PE anti-CD8 (clone 53-6.7; 1:50), PE anti-CD11b (clone M1/70; 1:100), PE anti-CD11c (clone HL3; 1:50), FITC anti-CD14 (clone rmC5-3; 1:50), FITC anti-CD19 (clone 1D3; 1:200), FITC anti-CD45 (clone 104; 1:100), PerCP anti-CD45 (clone 30-F11; 1:100), FITC anti-F4/80 (Caltag Laboratories, clone CI:A31; 1:100); PE anti-Gr-1 (clone RB6-8C5; 1:50); and PE anti-I-A/I-E (clone M5/114.15.2; 1:200).

\section{Statistics}

Data are expressed as mean \pm SEM, except as otherwise indicated. Student's 2-tailed nonpaired $t$ test or ANOVA and Bonferroni's multiple comparison tests were used to determine statistical significance (GraphPad Prism 4). Tumor-free survival was drawn using the KaplanMeier method and compared by the log-rank test. Differences were considered significant at $P<0.05$. 


\section{Study approval}

Mouse housing and all experimental animal procedures were performed in accordance with the guidelines established in the Principles of Laboratory Animal Care (directive 86/609/EEC) and approved by the Italian Ministry of Health. Specimens of PDAC and noncancerous pancreatic tissue were obtained upon informed consent from patients operated at University Hospitals Leuven. All tissue samples used for the TMAs were collected via standardized operative procedures approved by the Institutional Ethical Board of the European Institute of Oncology, and informed consent was obtained for all samples linked with clinical data.

\section{Acknowledgments}

We are grateful to Chiara Luise and Valentina Mattei for technical assistance; Monica Corada and Alicja Gruszka for technical advices;
Tania Roskams for providing tissue samples; Giovanni Bertalot for helping with vessel counting; and Pier Paolo Di Fiore for his support. This work was supported by research grants from the Association for International Cancer Research (AICR grant 10-0091), the Associazione Italiana Ricerca sul Cancro (AIRC grants 2214, 1378, and 5815), the Fondazione Cariplo (grant 2004-1587 and 20082375, the Fondazione Telethon (grant GGP04078), and the Italian Ministry of Health (Ricerca Finalizzata), and by a travel grant to E. Magrini from the European Society of Cardiology.

Address correspondence to: Ugo Cavallaro, Molecular Medicine Program, European Institute of Oncology, Via G. Ripamonti 435, I-20141 Milano, Italy. Phone: 39.02.9437.5165; E-mail: ugo. cavallaro@ieo.eu.
1. Ziyad S, Iruela-Arispe ML. Molecular mechanisms of tumor angiogenesis. Genes Cancer. 2011;2(12):1085-1096.

2. Ebos JM, Lee CR, Cruz-Munoz W, Bjarnason GA, Christensen JG, Kerbel RS. Accelerated metastasis after short-term treatment with a potent inhibitor of tumor angiogenesis. Cancer Cell. 2009;15(3):232-239.

3. Paez-Ribes M, et al. Antiangiogenic therapy elicits malignant progression of tumors to increased local invasion and distant metastasis. Cancer Cell. 2009;15(3):220-231.

4. Goel S, Wong AH, Jain RK. Vascular normalization as a therapeutic strategy for malignant and nonmalignant disease. Cold Spring Harb Perspect Med. 2012;2(3):a006486.

5. Moos M, Tacke R, Scherer H, Teplow D, Fruh K, Schachner M. Neural adhesion molecule L1 as a member of the immunoglobulin superfamily with binding domains similar to fibronectin. Nature. 1988;334(6184):701-703.

6. Rathjen FG, Schachner M. Immunocytological and biochemical characterization of a new neuronal cell surface component (L1 antigen) which is involved in cell adhesion. EMBO J. 1984;3(1):1-10.

7. Maness PF, Schachner M. Neural recognition molecules of the immunoglobulin superfamily: signaling transducers of axon guidance and neuronal migration. Nat Neurosci. 2007;10(1):19-26.

8. Kiefel H, et al. L1CAM: a major driver for tumor cell invasion and motility. Cell Adh Migr. 2012;6(4):374-384.

9. Pancook JD, Reisfeld RA, Varki N, Vitiello A, Fox RI, Montgomery AM. Expression and regulation of the neural cell adhesion molecule L1 on human cells of myelomonocytic and lymphoid origin. J Immunol. 1997;158(9):4413-4421.

10. Maddaluno L, et al. The adhesion molecule L1 regulates transendothelial migration and trafficking of dendritic cells. J Exp Med. 2009;206(3):623-635.

11. Issa $Y$, et al. Enhanced L1CAM expression on pancreatic tumor endothelium mediates selective tumor cell transmigration. JMol Med. 2009;87(1):99-112.

12. Potenta S, Zeisberg E, Kalluri R. The role of endothelial-to-mesenchymal transition in cancer progression. Br J Cancer. 2008;99(9):1375-1379.

13. Mazzone M, et al. Heterozygous deficiency of
PHD2 restores tumor oxygenation and inhibits metastasis via endothelial normalization. Cell. 2009;136(5):839-851.

14. Armulik A, Genove G, Betsholtz C. Pericytes: developmental, physiological, and pathological perspectives, problems, and promises. Dev Cell. 2011;21(2):193-215.

15. Gerhardt H, Betsholtz C. Endothelial-pericyte interactions in angiogenesis. Cell Tissue Res. 2003;314(1):15-23.

16. Poschl E, Schlotzer-Schrehardt U, Brachvogel B, Saito K, Ninomiya Y, Mayer U. Collagen IV is essential for basement membrane stability but dispensable for initiation of its assembly during early development. Development. 2004;131(7):1619-1628.

17. Lampugnani MG, et al. CCM1 regulates vascularlumen organization by inducing endothelial polarity. JCell Sci. 2010;123(pt 7):1073-1080.

18. Garlanda C, et al. Progressive growth in immunodeficient mice and host cell recruitment by mouse endothelial cells transformed by polyoma middle-sized $\mathrm{T}$ antigen: implications for the pathogenesis of opportunistic vascular tumors. Proc Natl Acad Sci USA. 1994;91(15):7291-7295.

19. Dejana E, Orsenigo F, Molendini C, Baluk P, McDonald DM. Organization and signaling of endothelial cell-to-cell junctions in various regions of the blood and lymphatic vascular trees. Cell Tissue Res. 2009;335(1):17-25.

20. Cavallaro U, Dejana E. Adhesion molecule signalling: not always a sticky business. Nat Rev Mol Cell Biol. 2011;12(3):189-197.

21. Medici D, Kalluri R. Endothelial-mesenchymal transition and its contribution to the emergence of stem cell phenotype. Semin Cancer Biol. 2012;22(5):379-384.

22. Cai X, Nomura-Kitabayashi A, Cai W, Yan J, Christoffels VM, Cai CL. Myocardial Tbx20 regulates early atrioventricular canal formation and endocardial epithelial-mesenchymal transition via Bmp2. Developmental biology. 2011;360(2):381-390.

23. Tusher VG, Tibshirani R, Chu G. Significance analysis of microarrays applied to the ionizing radiation response. Proc Natl Acad Sci US A. 2001;98(9):5116-5121.

24. Kamran MZ, Patil P, Gude RP. Role of STAT3 in cancer metastasis and translational advances.
Biomed Res Int. 2013;2013:421821.

25. Ara T, Declerck YA. Interleukin-6 in bone metastasis and cancer progression. Eur J Cancer. 2010;46(7):1223-1231.

26. Rawnaq T, et al. L1 is highly expressed in tumors of the nervous system: a study of over 8000 human tissues. J Surg Res. 2012;173(2):314-319.

27. Kaifi JT, et al. L1 (CD171) is highly expressed in gastrointestinal stromal tumors. Mod Pathol. 2006;19(3):399-406.

28 . Thies A, et al. Overexpression of the cell adhesion molecule $\mathrm{L} 1$ is associated with metastasis in cutaneous malignant melanoma. Eur J Cancer. 2002;38(13):1708-1716.

29. Dippel V, et al. Influence of L1-CAM expression of breast cancer cells on adhesion to endothelial cells. J Cancer Res Clin Oncol. 2013;139(1):107-121.

30. Voura EB, Ramjeesingh RA, Montgomery AM, Siu $\mathrm{CH}$. Involvement of integrin $\alpha(\mathrm{v}) \beta(3)$ and cell adhesion molecule L1 in transendothelial migration of melanoma cells. Mol Biol Cell. 2001;12(9):2699-2710.

31. Friedli A, et al. The soluble form of the cancerassociated L1 cell adhesion molecule is a pro-angiogenic factor. Int J Biochem Cell Biol. 2009;41(7):1572-1580.

32. Doberstein K, Pfeilschifter J, Gutwein P. The transcription factor PAX2 regulates ADAM10 expression in renal cell carcinoma. Carcinogenesis. 2011;32(11):1713-1723.

33. Hall H, Baechi T, Hubbell JA. Molecular properties of fibrin-based matrices for promotion of angiogenesis in vitro. Microvasc Res. 2001;62(3):315-326.

34. Blanco R, Gerhardt H. VEGF and Notch in tip and stalk cell selection. Cold Spring Harb Perspect Med. 2013;3(1):a006569.

35. Chen JC, Chang YW, Hong CC, Yu YH, Su JL. The role of the VEGF-C/VEGFRs axis in tumor progression and therapy. Int JMol Sci.2012;14(1):88-107.

36. Shrestha B, Ansari KI, Bhan A, Kasiri S, Hussain I, Mandal SS. Homeodomain-containing protein HOXB9 regulates expression of growth and angiogenic factors, facilitates tumor growth in vitro and is overexpressed in breast cancer tissue. FEBS J. 2012;279(19):3715-3726.

37. Padua D, et al. TGFbeta primes breast tumors for lung metastasis seeding through angiopoietinlike 4. Cell. 2008;133(1):66-77. 
38. Claesson-Welsh L, Welsh M. VEGFA and tumour angiogenesis. J Intern Med. 2013;273(2):114-127.

39. Huszar M, et al. Up-regulation of L1CAM is linked to loss of hormone receptors and E-cadherin in aggressive subtypes of endometrial carcinomas. J Pathol. 2010;220(5):551-561.

40. Kiefel H, et al. EMT-associated up-regulation of L1CAM provides insights into L1CAM-mediated integrin signalling and NF-KB activation. Carcinogenesis. 2012;33(10):1919-1929.

41. Schafer H, et al. TGF- $\beta 1$-dependent L1CAM expression has an essential role in macrophageinduced apoptosis resistance and cell migration of human intestinal epithelial cells. Oncogene. 2013;32(2):180-189.

42. Shtutman M, Levina E, Ohouo P, Baig M, Roninson IB. Cell adhesion molecule L1 disrupts E-cadherincontaining adherens junctions and increases scattering and motility of MCF7 breast carcinoma cells. Cancer Res. 2006;66(23):11370-11380.

43. Schafer $\mathrm{H}$, et al. Myofibroblast-induced tumorigenicity of pancreatic ductal epithelial cells is L1CAM dependent. Carcinogenesis. 2012;33(1):84-93.

44. De Craene B, Berx G. Regulatory networks defining EMT during cancer initiation and progression. Nat Rev Cancer. 2013;13(2):97-110.

45. Zeisberg M, Neilson EG. Biomarkers for epithelial-mesenchymal transitions. JClin Invest. 2009;119(6):1429-1437.
46. Middleton K, Jones J, Lwin Z, Coward JI. Interleukin-6: An angiogenic target in solid tumours. Crit Rev Oncol Hematol. 2014;89(1):129-139.

47. Scheller J, Garbers C, Rose-John S. Interleukin-6: From basic biology to selective blockade of pro-inflammatory activities. Semin Immunol. 2013;26(1):2-12.

48. Zarogoulidis P, et al. Interleukin-6 cytokine: a multifunctional glycoprotein for cancer. Immunome Res. 2013;9(62):16535.

49. Niu G, et al. Constitutive Stat 3 activity up-regulates VEGF expression and tumor angiogenesis. Oncogene. 2002;21(13):2000-2008.

50. Zhuang G, et al. Tumour-secreted miR-9 promotes endothelial cell migration and angiogenesis by activating the JAK-STAT pathway. EMBO J. 2012;31(17):3513-3523.

51. Son YS, et al. L1 cell adhesion molecule, a novel surface molecule of human embryonic stem cells, is essential for self-renewal and pluripotency. Stem Cells. 2011;29(12):2094-2099.

52. Deshpande N, Pysz MA, Willmann JK. Molecular ultrasound assessment of tumor angiogenesis. Angiogenesis. 2010;13(2):175-188.

53. Geninatti Crich S, et al. Magnetic resonance visualization of tumor angiogenesis by targeting neural cell adhesion molecules with the highly sensitive gadolinium-loaded apoferritin probe. Cancer Res. 2006;66(18):9196-9201.

54 . Grunberg J, et al. In vivo evaluation of $177 \mathrm{Lu}-$ and
67/64Cu-labeled recombinant fragments of antibody chCE7 for radioimmunotherapy and PET imaging of L1-CAM-positive tumors. Clin Cancer Res. 2005;11(14):5112-5120.

55. Weidle UH, Eggle D, Klostermann S. L1-CAM as a target for treatment of cancer with monoclonal antibodies. Anticancer Res. 2009;29(12):4919-4931.

56. Silveira CG, et al. L1 cell adhesion molecule as a potential therapeutic target in murine models of endometriosis using a monoclonal antibody approach. PLoS One. 2013;8(12):e82512.

57. Bazzoni G, Martinez-Estrada OM, Orsenigo F, Cordenonsi M, Citi S, Dejana E. Interaction of junctional adhesion molecule with the tight junction components ZO-1, cingulin, and occludin. J Biol Chem. 2000;275(27):20520-20526.

58. Irizarry RA, et al. Exploration, normalization, and summaries of high density oligonucleotide array probe level data. Biostatistics. 2003;4(2):249-264.

59. Schmittgen TD, Livak KJ. Analyzing real-time PCR data by the comparative C (T) method. Nat Protoc. 2008;3(6):1101-1108.

60. Mitchell TS, Bradley J, Robinson GS, Shima DT, Ng YS. RGS5 expression is a quantitative measure of pericyte coverage of blood vessels. Angiogenesis. 2008;11(2):141-151.

61. Zecchini S, et al. The differential role of $\mathrm{L} 1$ in ovarian carcinoma and normal ovarian surface epithelium. Cancer Res. 2008;68(4):1110-1118. 